Pacific

Journal of

Mathematics

GENERALIZED CARTAN TYPE S LIE ALGEBRAS IN CHARACTERISTIC 0 (II)

KAIMING ZHAO

Volume $192 \quad$ No. 2

February 2000 


\title{
GENERALIZED CARTAN TYPE S LIE ALGEBRAS IN CHARACTERISTIC 0 (II)
}

\author{
KAIMING ZHAO
}

\begin{abstract}
In this paper, we introduce a class of Lie algebras which are subalgebras of generalized Cartan type S Lie algebras of characteristic 0 . We determine the necessary and sufficient conditions for such Lie algebras to be simple. And we give all derivations of such simple Lie algebras.
\end{abstract}

\section{Introduction.}

This paper is a sequel to the paper [7] in which generalized Cartan type $\mathrm{S}$ Lie algebras $t^{z} S(A, T, \varphi)$ over a field $F$ of characteristic 0 were studied. We have tried to make this paper independent of other papers. So in Section 2, we give a description of relevant Lie algebras and some basic facts which will be used in this paper. In Section 3 we introduce a class of Lie algebras which are subalgebras of generalized Cartan type S Lie algebras, and determine the necessary and sufficient conditions for such Lie algebras to be simple. We give all derivations of such simple Lie algebras in Section 4.

This research was carried out during the author's visit to University of Wisconsin-Madison. He wishes to thank Professor J.M. Osborn for his hospitality and helpful discussions.

\section{Notations and related Lie algebras.}

In this section, for the convenience of the reader, we recall the relevant Lie algebra definitions and some basic facts which will be used later in this paper. Throughout this paper we assume that $F$ is a field of characteristic 0 , and that $A$ is a nonzero abelian group written additively.

\subsection{Generalized Witt algebras.}

Let $n$ be a positive integer, and $t_{1}, \ldots, t_{n}$ independent and commuting indeterminates over $F$. Denote by $P_{n}$ and $Q_{n}$ the polynomial algebra $F\left[t_{1}, \ldots, t_{n}\right]$, and the Laurent polynomial algebra $F\left[t_{1}^{ \pm 1}, \ldots, t_{n}^{ \pm 1}\right]$ respectively. By $W_{n}=W_{n}(F)$ we denote the Witt algebra, i.e., the Lie algebra of 
all formal vector fields

$$
\sum_{i=1}^{n} f_{i} \frac{\partial}{\partial t_{i}}
$$

with coefficients $f_{i} \in Q_{n}$. The bracket in $W_{n}$ is

$$
\left[f \frac{\partial}{\partial t_{i}}, g \frac{\partial}{\partial t_{j}}\right]=f \frac{\partial(g)}{\partial t_{i}} \frac{\partial}{\partial t_{j}}-g \frac{\partial(f)}{\partial t_{j}} \frac{\partial}{\partial t_{i}},
$$

where $f, g \in Q_{n}$, and $i, j \in\{1,2, \ldots, n\}$. The subalgebra $W_{n}^{+}=W_{n}^{+}(F)$ of $W_{n}$ consisting of all vector fields (2.1) with polynomial coefficients, i.e., $f_{i} \in P_{n}$, is known as the general Lie algebra, or the Lie algebra of Cartan type $W$. For more details, please refer to [13]. It is well known that $W_{n}$ and $W_{n}^{+}$are simple Lie algebras.

Let $T$ be a vector space over $F$. We denote by $F A$ the group algebra of $A$ over $F$. The elements $t^{x}, x \in A$, form a basis of this algebra, and the multiplication is defined by $t^{x} \cdot t^{y}=t^{x+y}$. We shall write 1 instead of $t^{0}$. The tensor product $W=F A \otimes_{F} T$ is a free left $F A$-module in the natural way. We denote an arbitrary element of $T$ by $\partial$ (to remind us of differential operators). For the sake of simplicity, we shall write $t^{x} \partial$ instead of $t^{x} \otimes \partial$. We now choose a pairing $\varphi: T \times A \rightarrow F$ which is $F$-linear in the first variable and additive in the second one. For convenience we shall also use the following notations:

$$
\varphi(\partial, x)=\langle\partial, x\rangle=\partial(x)
$$

for arbitrary $\partial \in T$ and $x \in A$. $W$ becomes a Lie algebra under the following bracket:

$$
\left[t^{x} \partial_{1}, t^{y} \partial_{2}\right]:=t^{x+y}\left(\partial_{1}(y) \partial_{2}-\partial_{2}(x) \partial_{1}\right)
$$

for arbitrary $x, y \in A$ and $\partial_{1}, \partial_{2} \in T$. We refer to this algebra $W=$ $W(A, T, \varphi)$ as a generalized Witt algebra.

The subspaces $W_{x}=t^{x} T, x \in A$, define an $A$-gradation of $W$, i.e., $W$ is the direct sum of the $W_{x}$ 's, and $\left[W_{x}, W_{y}\right] \subset W_{x+y}$ for all $x, y \in A$.

It follows from (2.2) that $\operatorname{ad}(\partial)$ acts on $W_{x}$ as a scalar $\partial(x)$. Hence each $\partial \in T$ is ad-semisimple, and $T$ is a torus (i.e., an abelian subalgebra consisting of ad-semisimple elements). In fact $T$ is the only maximal torus of $W$ (see [3, Lemma 4.1]). Kawamoto proved in [11] that the Lie algebra $W=W(A, T, \varphi)$ is simple if and only if $A \neq 0$ and $\varphi$ is nondegenerate in the sense that the conditions

$$
\langle\partial, x\rangle=0, \forall \partial \in T \Rightarrow x=0
$$

and

$$
\langle\partial, x\rangle=0, \forall x \in A \Rightarrow \partial=0
$$

hold. 
Note that (2.3) implies that $A$ is torsion free. This implies that $F A$ is an integral domain and it implies that the invertible elements of $F A$ have the form $a t^{x}$, where $a \in F^{*}, x \in A$.

There is a natural structure of a left $W$-module on $F A$, namely the structure is such that

$$
t^{x} \partial \cdot t^{y}=\partial(y) t^{x+y}
$$

for $x, y \in A$ and $\partial \in T$. Also we have the natural left $F A$-module structure on $W$. These two module structures are related by the identity

$$
[f u, g v]=f(u \cdot g) v-g(v \cdot f) u+f g[u, v]
$$

where $f, g \in F A$ and $u, v \in W$ are arbitrary. The $W$-module structure on $F A$ gives rise to a homomorphism

$$
W \rightarrow \operatorname{Der}(F A)
$$

because each $w \in W$ acts on $F A$ as a derivation. Clearly (2.7) is also a homomorphism of $F A$-modules. For more details about $W(A, T, \varphi)$, please refer to $[\mathbf{3}]$.

\subsection{Generalized Cartan type W Lie algebras.}

Suppose that $W=W(A, T, \varphi)$ denotes a simple generalized Witt algebra. Let $I$ be an index set, $d: I \rightarrow T$ an injective map, and write $d_{i}=d(i)$ for $i \in I$. We say that $d$ is admissible if the following two conditions hold:

(Ind) $d_{i}, i \in I$, are linearly independent;

(Int) $\quad d_{i}(A)=\mathbf{Z}$ for all $i \in I$.

We assume throughout that an admissible $d$ has been fixed. We set

$$
\begin{aligned}
A_{d}^{+} & =\left\{x \in A: d_{i}(x) \geq 0, \forall i \in I\right\}, \\
A_{d}^{0} & =\left\{x \in A: d_{i}(x)=0, \forall i \in I\right\}, \\
A_{d, i} & =\left\{x \in A: d_{i}(x)=-1 ; d_{j}(x) \geq 0, \forall j \in I \backslash\{i\}\right\}, \\
A_{d, i}^{\#} & =\left\{x \in A: d_{i}(x)=-1 ; d_{j}(x)=0, \forall j \in I \backslash\{i\}\right\}, \\
A_{d} & =A_{d}^{+} \cup\left(\cup_{i \in I} A_{d, i}\right) .
\end{aligned}
$$

We now introduce some subalgebras of $W$ :

$$
\begin{aligned}
W_{d}^{+} & =\sum_{x \in A_{d}^{+}} W_{x} ; \\
W_{d, i} & =\left(\sum_{x \in A_{d, i}} F t^{x}\right) \cdot d_{i}, i \in I ;
\end{aligned}
$$


and

$$
W_{d}=W_{d}(A, T, \varphi)=W_{d}^{+}+\sum_{i \in I} W_{d, i} .
$$

We also introduce the subalgebra $F A_{d}^{+}$of $F A$, which is the span of all elements $t^{x}$ with $x \in A_{d}^{+}$. Since $W$ is a left $F A$-module, we can view $W$ also as a left $F A_{d}^{+}$-module. Then it is easy to see that the subspaces $W_{d}^{+}$and $W_{d}$ are $F A_{d}^{+}$-submodules of $W$.

By restricting the action of $W$ on $F A$, we can view $F A$ as a left $W_{d^{-}}$ module, and then $F A_{d}^{+}$is a $W_{d}$-submodule of $F A$. When $d$ is fixed, and there is no danger of confusion, we shall write

$$
A^{+}, A_{i}, A_{i}^{\#}, W^{+}, W_{i}, F A^{+}
$$

instead of

$$
A_{d}^{+}, A_{d, i}, A_{d, i}^{\#}, W_{d}^{+}, W_{d, i}, F A_{d}^{+},
$$

respectively. The following Theorem is proved in [5].

Theorem 2.1. The Lie algebra $W_{d}$ is simple if and only if the following conditions hold:

(i) if $\partial \in T$ and $\partial(x)=0$ for all $x \in A_{d}$, then $\partial=0$;

(ii) if $x \in A_{d}$, then $d_{i}(x)=0$ for almost all $i \in I$;

(iii) $A_{i}^{\#} \neq \emptyset$ for all $i \in I$.

The simple Lie algebra $W_{d}$ is called an algebra of generalized Cartan type $W$. For more details on the Lie algebra $W_{d}$, please refer to the papers [5] and $[\mathbf{1 2}]$.

\subsection{Generalized Cartan type S Lie algebras.}

It is well known that the classical divergence Div: $W_{n} \rightarrow F\left[t_{1}^{ \pm 1}, \ldots, t_{n}^{ \pm 1}\right]$ maps $\sum_{i=1}^{n} f_{i} \frac{\partial}{\partial t_{i}}$ to $\sum_{i=1}^{n} \frac{\partial\left(f_{i}\right)}{\partial t_{i}}$. The kernel $\tilde{S}_{n}$ of Div is a subalgebra of $W_{n}$. The algebra $S_{n}=\left(\tilde{S}_{n}\right)^{\prime \prime}$ and $S_{n}^{+}=S_{n} \cap W_{n}^{+}$are called Lie algebras of Cartan type $\mathrm{S}$.

Suppose that $W=W(A, T, \varphi)$ is a simple generalized Witt algebra. The divergence div: $W \rightarrow F A$ is the $F$-linear map such that

$$
\operatorname{div}\left(t^{x} \partial\right)=\partial(x) t^{x}
$$

holds for all $x \in A$ and $\partial \in T$. It has the following two properties:

$$
\operatorname{div}(f w)=f \operatorname{div}(w)+w \cdot f
$$

and

$$
\operatorname{div}[u, v]=u \cdot \operatorname{div}(v)-v \cdot \operatorname{div}(u)
$$


where $u, v, w \in W$ and $f \in F A$ are arbitrary. The latter property shows that div is a derivation of $W$ with values in the $W$-module $F A$. Since $\operatorname{div}: W \rightarrow F A$ is a derivation of degree 0 , its kernel $\tilde{S}:=\operatorname{ker}(\operatorname{div})$ is a homogeneous subalgebra of $W$ :

$$
\tilde{S}=\oplus_{x \in A} \tilde{S}_{x}, \tilde{S}_{x}:=\tilde{S} \cap W_{x} .
$$

For $x \in A$ define the $F$-linear function $\hat{x}: T \rightarrow F$ by $\hat{x}(\partial)=\partial(x)$. The condition (2.3) shows that, if $T^{*}$ is the dual space of $T$, the $\mathbf{Z}$-linear map $A \rightarrow T^{*}$ sending $x \mapsto \hat{x}$ is injective. If $T_{x}:=\operatorname{ker}(\hat{x})$, then we have $\tilde{S}_{x}=t^{x} T_{x}$. Hence $\tilde{S}_{0}=W_{0}=T$ and, for $x \neq 0, \tilde{S}_{x}$ is a hyperplane of $W_{x}$. In particular, if $\operatorname{dim} T=1$, then $\tilde{S}=T$. To avoid trivialities, we shall assume always that $\operatorname{dim} T>1$.

Let $\bar{S}:=(\tilde{S})^{\prime}$ be the derived algebra of $\tilde{S}$. Note that the notation here is different from that in $[\mathbf{7}]$. We know (see [7]) that

$$
\bar{S}=\oplus_{x \neq 0} \tilde{S}_{x} .
$$

More generally, the subspaces $t^{z} \tilde{S}, z \in A$, are subalgebras of $W$ and their derived algebras are given by

$$
\left(t^{z} \tilde{S}\right)^{\prime}=t^{z} \bar{S}=\sum_{x \neq z} t^{x} T_{x-z} .
$$

If $\operatorname{dim} T \geq 3$, all the subalgebras $t^{z} \bar{S}$ are simple. If $\operatorname{dim} T=2$, then $\bar{S}$ itself is simple while the shifted algebras $t^{z} \bar{S}, z \neq 0$, are not. Their derived algebras

$$
\left(t^{z} \bar{S}\right)^{\prime}=\sum_{x \neq z, 2 z} t^{x} T_{x-z}, z \neq 0
$$

are simple.

We shall refer to the subalgebras $S(A, T, \varphi, z):=t^{z} \bar{S}$ if $\operatorname{dim} T \geq 3$, and $S(A, T, \varphi, z):=\left(t^{z} \bar{S}\right)^{\prime}$ if $\operatorname{dim} T=2$, as Lie algebras of generalized Cartan type $S$. The Lie algebras $S(A, T, \varphi, z)$ have the $A$-gradation:

$$
S(A, T, \varphi, z)= \begin{cases}\oplus_{x \in A \backslash\{z\}} t^{x} T_{x-z}, & \text { if } \operatorname{dim} T>2, \\ \oplus_{x \in A \backslash\{z, 2 z\}} t^{x} T_{x-z}, & \text { if } \operatorname{dim} T=2 .\end{cases}
$$

These algebras were studied in papers: [6] when $\operatorname{dim} T=2$ and $z=0,[4]$ when $\operatorname{dim} T=2$ and $z \neq 0$, and [7] when $\operatorname{dim} T \geq 3$.

\subsection{Generalized Block algebras.}

We shall denote by $\operatorname{Hom}(A, F)$ the $F$-vector space of all additive (i.e., Zlinear) maps $A \rightarrow F$. We now fix an additive map $\alpha: A \rightarrow F$ and a skew-symmetric bi-additive map $\varphi: A \times A \rightarrow F$.

Let $L=L(A, \alpha, \varphi)$ be the vector space over $F$ having a basis consisting of all symbols $e_{x}, x \in A$. We make $L$ into a (non-associative) algebra over 
$F$ by defining $F$-bilinear multiplication $L \times L \rightarrow L$ by

$$
\left[e_{x}, e_{y}\right]=f(x, y) e_{x+y}, x, y \in A,
$$

where

$$
f(x, y)=\varphi(x, y)+\alpha(x-y) .
$$

If $\alpha=0$, then $L$ is a Lie algebra. These Lie algebras were studied in [8]. It was shown by Albert and Frank [1] that, under the assumption that $\alpha \neq 0$, $L$ is a Lie algebra if and only if there exists another additive map $\beta: A \rightarrow F$ such that $\varphi=\alpha \wedge \beta$, i.e.,

$$
\varphi(x, y)=\alpha(x) \beta(y)-\beta(x) \alpha(y) .
$$

We shall assume throughout that such a $\beta$ exists, i.e. that $L$ is a Lie algebra. We also assume that

$$
K_{\alpha} \cap K_{\beta}=0,
$$

where $K_{\mu}$ denotes the kernel of $\mu$ for any additive map $\mu: A \rightarrow F$. Let $L^{2}=[L, L]$ be the derived subalgebra of $L$ and $Z$ the center of $L$. The Lie algebra $\mathcal{L}=L^{2} / Z$ is simple [4, Theorem 2.5], and we shall also write $\mathcal{L}(A, \alpha, \varphi)$ for $\mathcal{L}$.

We refer to the Lie algebras $\mathcal{L}=\mathcal{L}(A, \alpha, \varphi)$ as generalized Block algebras.

Suppose that $\beta \in \operatorname{Hom}(A, F)$ can be chosen so that (2.15) holds and

$$
\beta(A)=\mathbf{Z} .
$$

We now define the subset $A_{\beta} \subset A$ by

$$
A_{\beta}=\{x \in A: \beta(x) \geq-1\},
$$

and denote by $L_{\beta}$ the subspace of $L$ with a basis consisting of all $e_{x}$ with $x \in A_{\beta}$. It follows that $L_{\beta}$ is a subalgebra of $L$.

We shall denote by $\mathcal{L}_{\beta}$ or $\mathcal{L}_{\beta}(A, \alpha, \varphi)$ the quotient algebra $L_{\beta} / Z$. The Lie algebra $\mathcal{L}_{\beta}$ is simple. For more details about $\mathcal{L}(A, \alpha, \varphi)$ and $\mathcal{L}_{\beta}(A, \alpha, \varphi)$, please refer to $[4]$ and $[8]$.

\section{Lie algebra $S_{d}(A, T, \varphi, z)$.}

Now we are ready to introduce our main object, the Lie algebra $S_{d}(A, T, \varphi, z)$. We assume that a generalized Witt algebra $W=W(A, T, \varphi)$, a generalized Cartan type W Lie algebra $W_{d}=W_{d}(A, T, \varphi) \subset W$, a generalized Cartan type $\mathrm{S}$ Lie algebra $S(A, T, \varphi, z) \subset W$ are given, and we also assume throughout the paper that all of these algebras are simple. Our hypotheses here imply that $\operatorname{dim} T \geq 2$. We define

$$
S_{d}(A, T, \varphi, z):=W_{d} \cap S(A, T, \varphi, z) .
$$


Then $S_{d}=S_{d}(A, T, \varphi, z)$ is a subalgebra of $W$. We shall also call the algebra $S_{d}$ a generalized Cartan type $\mathrm{S}$ Lie algebra. It is clear that $S_{d}$ has an $A$ gradation with the following components of degree $x \in A$ :

$$
\left(S_{d}\right)_{x}= \begin{cases}0, & \text { for } x=z \\ \left(t^{x} T_{x-z}\right) \cap W_{d}, & \text { for } x \in A \backslash\{z\}\end{cases}
$$

if $\operatorname{dim} T>2$,

$$
\left(S_{d}\right)_{x}= \begin{cases}0, & \text { for } x=z \text { or } x=2 z \\ \left(t^{x} T_{x-z}\right) \cap W_{d}, & \text { for } x \in A \backslash\{z, 2 z\}\end{cases}
$$

if $\operatorname{dim} T=2$. It follows that $\left(S_{d}\right)_{x}=0$ for all $x \notin A_{d}$.

For convenience, by $\left\langle U_{1}\right\rangle$ we denote the subspace of the vector space $V$ generated by $U_{1} \subset V$.

Theorem 3.1. Suppose that the Lie algebras $W=W(A, T, \varphi), W_{d}=$ $W_{d}(A, T, \varphi) \subset W$, and $S=S(A, T, \varphi, z) \subset W$ are given, and that all of them are simple. Then $S_{d}(A, T, \varphi, z)$ is simple if and only if the following conditions hold:

(a) $d_{i}(z)=-1$ for all $i \in I$;

(b) I is finite.

Proof. For simplicity we write $L=S_{d}(A, T, \varphi, z)$, so $L_{x}=\left(S_{d}\right)_{x}$ for $x \in A$.

$(\Rightarrow)$ Suppose that $L$ is simple. We shall first show that (a) is true. For contradiction we assume that there exists an element in $I$, say 1 , such that $d_{1}(z) \neq-1$. If $t^{x} d_{1} \in W_{1} \cap L$ (for definition of $W_{1}$ see Section 2.2), we know that $d_{1}(x)=-1$ and $d_{1}(x-z)=0$, so $-1=d_{1}(x)=d_{1}(z) \neq-1$. It is a contradiction. Then $W_{1} \cap L=0$, i.e., $L_{x}=0$ for all $x \in A_{1}$. Theorem 2.1(iii) assures that we can choose $y \in A$ with $-y \in A_{1}^{\#}$. So $y \in A_{d}^{+}$. If $z \neq y$, then $L_{y}=t^{y} T_{y-z} \neq 0$. Let $J=\oplus_{x: d_{1}(x)>1} L_{x}$. If $z=y$, we see that $L_{3 y} \neq 0$, let $J=\oplus_{x}: d_{1}(x)>3 L_{x}$. It is easy to verify that $J$ is a nonzero proper ideal of $L$ in both cases, which contradicts the simplicity of $L$. Consequently (a) holds.

On the other side we have $-z \in A_{d}^{+}$. From Theorem 2.1(ii) it follows that $I$ is finite. So (b) is true.

$(\Leftarrow)$ Suppose (a) and (b) hold. We write $I=\{1,2, \cdots, n\}$. We know that $L_{x} \neq 0$ if and only if $x \in A_{d} \backslash\{z\}$. Fix $\left\{u_{1}, u_{2}, \cdots, u_{n}\right\} \subset A$ such that $d_{i}\left(u_{j}\right)=\delta_{i, j}$. Let $A(d)=\mathbf{Z} u_{1} \oplus \mathbf{Z} u_{2} \oplus \cdots \oplus \mathbf{Z} u_{n}$, and $A^{\prime}(d)=\{x \in$ $\left.A \mid d_{i}(x)=0 \forall i \in I\right\}$. Then it follows from (b) that $A=A(d) \oplus A^{\prime}(d)$.

Case 1. Suppose that $\operatorname{dim} T=2$ and $|I|=1$, say $I=\{1\}$. Choose $d_{2} \in T$ such that $T=F d_{1} \oplus F d_{2}$. Denote $e_{x}=t^{x}\left(d_{1}(x-z) d_{2}-d_{2}(x-z) d_{1}\right)$ for 
$x \in A_{d}$. It follows that $\left\{e_{x} \mid x \in A_{d} \backslash\{z\}\right\}$ is a basis of $L=S_{d}(A, T, \varphi, z)$. For any $x, y \in A_{d}$ we have

$$
\begin{aligned}
{\left[e_{x}, e_{y}\right]=} & {\left[t^{x}\left(d_{1}(x-z) d_{2}-d_{2}(x-z) d_{1}\right), t^{y}\left(d_{1}(y-z) d_{2}-d_{2}(y-z) d_{1}\right)\right] } \\
= & t^{x+y}\left(\left(d_{1}(x-z) d_{2}(y)-d_{2}(x-z) d_{1}(y)\right)\right. \\
& \cdot t^{x}\left(d_{1}(x-z) d_{2}-d_{2}(x-z) d_{1}\right) \\
- & \left(d_{1}(y-z) d_{2}(x)-d_{2}(y-z) d_{1}(x)\right) \\
& \left.\cdot t^{y}\left(d_{1}(y-z) d_{2}-d_{2}(y-z) d_{1}\right)\right) \\
= & t^{x+y}\left(d_{1}(x-z) d_{2}(y-z)-d_{2}(x-z) d_{1}(y-z)\right) \\
& \cdot\left(d_{1}(x+y-z) d_{2}-d_{2}(x+y-z) d_{1}\right) \\
= & \left|\begin{array}{ll}
d_{1}(x-z) & d_{1}(y-z) \\
d_{2}(x-z) & d_{2}(y-z)
\end{array}\right| e_{x+y} .
\end{aligned}
$$

Denote $\alpha(x)=-d_{2}(x)-d_{2}(z) d_{1}(x), \beta(x)=d_{1}(x)$ for $x, y \in A$. It is clear that $\beta(A)=\mathbf{Z}$. By Section 2.4 we know that $L \simeq \mathcal{L}_{\beta}(A, \alpha, \beta)$. Consequently $L$ is simple.

Case 2. Suppose that $\operatorname{dim} T=2$ and $|I|=2$, say $I=\{1,2\}$. Since $\varphi$ is nondegenerate, we get that $A=A(d)=d_{1}(A) \otimes d_{2}(A) \simeq \mathbf{Z} \otimes \mathbf{Z}$. We may assume that $d_{i}(x)=x_{i}$ for $x=\left(x_{1}, x_{2}\right) \in \mathbf{Z} \otimes \mathbf{Z}$. Thus $A_{d}=$ $\left(\left(\mathbf{Z}_{+}-1\right) \otimes\left(\mathbf{Z}_{+}-1\right)\right) \backslash\{(-1,-1)\}$. Same as Case 1 , we define $e_{x}, x \in A_{d}$. Then we also have

$$
\left[e_{x}, e_{y}\right]=\left|\begin{array}{ll}
d_{1}(x-z) & d_{1}(y-z) \\
d_{2}(x-z) & d_{2}(y-z)
\end{array}\right| e_{x+y}
$$

i.e.,

$$
\left[e_{x}, e_{y}\right]=\left|\begin{array}{ll}
x_{1}+1 & y_{1}+1 \\
x_{2}+1 & y_{2}+1
\end{array}\right| e_{x+y}, \forall x, y \in A_{d}
$$

It is well known that the Lie algebra $S_{2}^{+} \subset W_{2}^{+}$has basis $\left\{t_{1}^{x_{1}} t_{2}^{x_{2}}\left(t_{2} \frac{\partial}{\partial t_{1}}-\right.\right.$ $\left.\left.t_{1} \frac{\partial}{\partial t_{2}}\right) \mid\left(x_{1}, x_{2}\right) \in A_{d}\right\}$. It is easy to verify that the following linear map is an isomorphism of Lie algebras:

$$
S_{d} \rightarrow S_{2}^{+}, e_{x} \mapsto t_{1}^{x_{1}} t_{2}^{x_{2}}\left(t_{2} \frac{\partial}{\partial t_{1}}-t_{1} \frac{\partial}{\partial t_{2}}\right) .
$$

Thus $S_{d}$ is also simple in this case.

Case 3. Suppose that $\operatorname{dim} T>2$.

If $|I|=\operatorname{dim} T=n$, as we did in Case 2 we can deduce that $L \simeq S_{n}^{+}$, the special algebra of rank $n$. Thus in this case $S_{d}$ is simple. Next we assume that $\operatorname{dim} T>|I|=n>0$. 
Let $J$ be a nonzero ideal of $L$. It is suffices to show that $J=L$. Choose a nonzero element $u \in J$, say

$$
u=\sum_{i=1}^{m} t^{x_{i}} \partial_{i}, x_{i} \in A_{d} \backslash\{z\}, \partial_{i} \in T_{x_{i}-z}
$$

with $m$ is minimal. Then $x_{1}, \cdots, x_{m}$ are distinct and $\partial_{i} \neq 0$.

Claim 1. We have that $m=1$.

Otherwise we suppose $m>1$. Since $T_{z} \subset L$, from the minimality of $m$ it follows that

$$
\left.\left(\hat{x}_{1}\right)\right|_{T_{z}}=\left.\left(\hat{x}_{2}\right)\right|_{T_{z}}=\cdots=\left.\left(\hat{x}_{m}\right)\right|_{T_{z}} .
$$

Thus

$$
\hat{x}_{i}-\hat{x}_{j} \in F \hat{z}, \forall i, j \in\{1,2, \cdots, m\} .
$$

Subclaim. We can choose such an element $u$ in (3.1) such that $d_{1}\left(x_{1}\right)=$ -1 .

Suppose $d_{1}\left(x_{1}\right) \geq 0$. If $\partial_{1} \in F d_{1}$, let $y_{1} \in A_{1}^{\#}$, then

$$
u^{\prime}=\left[t^{y_{1}} d_{1}, u\right]=\sum_{i=1}^{m} t^{y_{1}+x_{i}}\left(d_{1}\left(x_{i}\right) \partial_{i}-\partial_{i}\left(y_{1}\right) d_{1}\right) \in J \backslash\{0\}
$$

and $d_{1}\left(y_{1}+x_{1}\right)<d_{1}\left(x_{1}\right)$. If $\partial_{1} \notin F d_{1}$, then $A_{d, 1} \not \subset \operatorname{ker}\left(\partial_{1}\right)$, Otherwise we can show that $\partial_{1}\left(A_{d}\right)=0$, it is impossible. We choose $y_{1} \in A_{d, 1} \backslash \operatorname{ker}\left(\partial_{1}\right)$. We deduce that

$$
u^{\prime}=\left[t^{y_{1}} d_{1}, u\right]=\sum_{i=1}^{m} t^{y_{1}+x_{i}}\left(d_{1}\left(x_{i}\right) \partial_{i}-\partial_{i}\left(y_{1}\right) d_{1}\right) \in J \backslash\{0\},
$$

and also $d_{1}\left(y_{1}+x_{1}\right)<d_{1}\left(x_{1}\right)$. After finitely many steps of this kind process, we get a nonzero element $u$ in (3.1) such that $d_{1}\left(x_{1}\right)=-1$. Our subclaim follows.

Without loss of generality we may assume that $\partial_{1}=d_{1}$. From (3.2) it follows that there exists $\lambda_{i} \in F^{*}$ such that

$$
\hat{x}_{i}=\lambda_{i} \hat{z}+\hat{x}_{1}, \forall i \in\{2, \cdots, m\} .
$$

Since $x_{i} \in A_{d}$, we have $d_{1}\left(x_{i}\right) \geq-1$, then $-\lambda_{i} \in \mathbf{N}$, the set of natural numbers. Thus $d_{1}\left(x_{2}\right) \geq 0$. If $\partial_{2} \in F d_{1}$, then

$$
\left[t^{x_{1}} d_{1}, t^{x_{2}} \partial_{2}\right]=t^{x_{1}+x_{2}}\left(d_{1}\left(x_{i}\right) \partial_{i}-\partial_{i}\left(y_{1}\right) d_{1}\right) \neq 0 .
$$

It follows that

$$
u^{\prime}=\left[t^{x_{1}} d_{1}, u\right]=\sum_{i=2}^{m} t^{x_{1}+x_{i}}\left(d_{1}\left(x_{i}\right) \partial_{i}-\partial_{i}\left(y_{1}\right) d_{1}\right) \in J \backslash\{0\} .
$$


This contradicts the minimality of $m$. Consequently $\partial_{2} \notin F d_{1}$. Choose $z_{1} \in$ $A_{d, 1} \backslash \operatorname{ker}\left(\partial_{2}\right)$, then $\left[t^{x_{1}} d_{1}, t^{z_{1}} d_{1}\right]=0$ and $\left[t^{z_{1}} d_{1}, t^{x_{2}} \partial_{2}\right]=t^{z_{1}+x_{2}}\left(d_{1}\left(x_{2}\right) \partial_{2}-\right.$ $\left.\partial_{2}\left(z_{1}\right) d_{1}\right) \neq 0$. Thus

$$
u^{\prime}=\left[t^{z_{1}} d_{1}, u\right]=\sum_{i=2}^{m} t^{z_{1}+x_{i}}\left(d_{1}\left(x_{i}\right) \partial_{i}-\partial_{i}\left(z_{1}\right) d_{1}\right) \in J \backslash\{0\} .
$$

It again contradicts the minimality of $m$. Therefore $m=1$. Claim 1 follows.

Claim 2. We have $T_{z} \subset J$.

From Claim 1 we know that there exists a nonzero element $t^{x} \partial_{0} \in J$, where $x \in A_{d}, \partial_{0} \in T_{x-z}$.

Subcase 1. Suppose $x \in A_{d, i}^{\#}$ for some $i \in I$, say $x \in A_{d, 1}^{\#}$. Then $\partial_{0} \in F d_{1}$. Thus $t^{x} d_{1} \in J$. For any $\partial \in T_{-x-z}$, from

$$
\left[t^{x} d_{1}, t^{-x} \partial\right]=\partial-\partial(x) d_{1} \in J
$$

and $d_{1} \notin T_{-x-z}$, we know that $\left\langle d_{1}, \partial-\partial(x) d_{1} \mid \partial \in T_{-x-z}\right\rangle=T$. Hence $\left\langle\partial-\partial(x) d_{1} \mid \partial \in T_{-x-z}\right\rangle=T_{-z}=T_{z}$. therefore $T_{z} \subset J$.

Subcase 2. Suppose $x \in A_{d}^{+}$. Define $d(x):=\sum_{i=1}^{n} d_{i}(x)$. If $d_{1}(x)>0$, choose $y_{1} \in A_{d, 1}^{\#}$. Since $d_{1}(x-z) \neq 0$ and $\partial_{0}(x-z)=0$, we know that $d_{1}$ and $\partial_{0}$ are linearly independent. From the computation

$$
\left[t^{y_{1}} d_{1}, t^{x} \partial_{0}\right]=t^{x+y_{1}}\left(d_{1}(x) \partial_{0}-\partial_{0}\left(y_{1}\right) d_{1}\right) \neq 0,
$$

we get a nonzero element $t^{x+y_{1}}\left(d_{1}(x) \partial_{0}-\partial_{0}\left(y_{1}\right) d_{1}\right) \in J$ with $d\left(x+y_{1}\right)=$ $d(x)-1$. By repeatedly using this method, after finitely many steps we deduce that there exists a nonzero element $t^{y} \partial \in J$ with $y \in A_{d}^{0}$. If $A_{d, i}^{\#} \subset$ $\operatorname{ker}(\partial)$ for all $i \in I$, we infer that $\partial\left(A_{d}\right)=0$. It contradicts Theorem 2.1(i). Thus there exists an $i \in I$ such that $A_{d, i}^{\#} \not \subset \operatorname{ker}(\partial)$, say $A_{d, 1}^{\#} \not \subset \operatorname{ker}(\partial)$. Choose $z_{1} \in A_{d, 1}^{\#} \backslash \operatorname{ker}(\partial)$, then

$$
\left[t^{z_{1}} d_{1}, t^{y} \partial\right]=-t^{z_{1}+y} \partial\left(z_{1}\right) d_{1} \in J \backslash\{0\} .
$$

By Subcase 1 we obtain again that $T_{z} \subset J$. Similarly $T_{z} \subseteq J$ for $x \in A_{d, i}$. Thus Claim 2 is proved.

If $x \in A_{d}$ and $\hat{x} \notin F \hat{z}$, then $T_{z} \not \subset$ ker $\hat{x}$. Choose $\partial \in T_{z} \backslash \operatorname{ker} \hat{x}$. From $\left[\partial, t^{x} \partial^{\prime}\right]=\partial(x) t_{x} \partial^{\prime} \in J$ we know that $t^{x} T_{x-z} \subset J$.

If $y \in A_{d} \backslash\{0\}$ and $\hat{y} \in F \hat{z}$, it follows that $y=-k z$ for some positive integer $k$. Since $\left\{\hat{x} \mid x \in A_{d, 1}^{\#}\right\} \not \subset F \hat{z}$, we choose $x_{1} \in A_{d, 1}^{\#}$ with $\hat{x}_{1} \notin F \hat{z}$. Since $y \neq z$, then $d_{i}(y)>0$ for all $i \in I$. Note that $t^{y-x_{1}} T_{y-x_{1}-z} \subset J$. Then, for $t^{x_{1}} d_{1}, t^{y-x_{1}} \partial_{1} \in L$, we have

$$
\left[t^{y-x_{1}} \partial_{1}, t^{x_{1}} d_{1}\right]=-t^{y}\left(d_{1}\left(y-x_{1}\right) \partial_{1}-\partial_{1}(x) d_{1}\right) \in J .
$$


Because $\left\langle d_{1}, d_{1}\left(y-x_{1}\right) \partial_{1}-\partial_{1}(x) d_{1} \mid \partial_{1} \in T_{y-x_{1}-z}\right\rangle=T$. Then $\left\langle d_{1}\left(y-x_{1}\right) \partial_{1}-\right.$ $\partial_{1}(x) d_{1}\left|\partial_{1} \in T_{y-x_{1}-z}\right\rangle=T_{y-z}=T_{z}$, hence $L_{y} \in J$. Therefore $J=L$. This completes the proof of this theorem.

Note that $z \notin A_{d}$ if $|I|>1$, and $z \in A_{d}$ if $|I|=1$. The following corollary follows directly from the above theorem.

Corollary 3.2. Suppose that $S_{d}=S_{d}(A, T, \varphi, z)$ is simple. Then $\left(S_{d}\right)_{x} \neq 0$ if and only if $x \in A_{d} \backslash\{z\}$.

\section{Derivations of $S_{d}(A, T, \varphi, z)$.}

In this section we assume that the Lie algebra $S_{d}(A, T, \varphi, z)$ is simple, and we shall determine all the derivations of $S_{d}(A, T, \varphi, z)$. From the proof of Theorem 3.1 we know that:

(a) If $I=\emptyset, S_{d}(A, T, \varphi, z)=S(A, T, \varphi, z)$, which was thoroughly studied in $[7]$;

(b) If $|I|=\operatorname{dim} T=n, S_{d}(A, T, \varphi, z) \simeq S_{n}^{+}$, which was studied in many references, for example [12];

(c) If $\operatorname{dim} T=2$ and $|I|=1, S_{d}(A, T, \varphi, z) \simeq \mathcal{L}_{\beta}(A, \alpha, \beta)$ for some suitable $\alpha, \beta \in \operatorname{hom}(A, F)$, which was thoroughly studied in [8].

So from now on we always assume that $0<|I|<\operatorname{dim} T$ and $\operatorname{dim} T \geq 3$. Write $L=S_{d}(A, T, \varphi, z), I=\{1,2, \cdots, n\}$ and $S_{d}^{+}=\sum_{x \in A_{d}^{+}}\left(S_{d}\right)_{x}$. Recall that $L$ has an $A$-gradation with the following components of degree $x \in A$ :

$$
L_{x}= \begin{cases}0, & \text { for } x=z \\ \left(t^{x} T_{x-z}\right) \cap W_{d}, & \text { for } x \in A \backslash\{z\} .\end{cases}
$$

A derivation $D$ of $L$ is called homogeneous of degree $x \in A$ if $D\left(L_{y}\right) \subset$ $L_{x+y}$ for all $y \in A$.

From direct computation we can easily obtain the following lemma.

Lemma 4.1. Every $D \in \operatorname{Der}(L)$ has the form

$$
D=\sum_{y \in A} D_{y}
$$

where $D_{y}$ is a derivation of $L$ of degree $y$, such that for each $u \in L$ there are only finitely many $y \in A$ with $D_{y}(u) \neq 0$.

First we construct some derivations of $S_{d}$. For any additive function $\mu: A \rightarrow F$ the linear map

$$
D_{\mu}(X)=\mu(x) X, \forall X \in\left(S_{d}\right)_{x}
$$

is a derivation of $S_{d}$ of degree 0 .

The following Lemmas 4.2-4.5 will be useful in the sequel. 
Lemma 4.2. Let $x, y \in A \backslash\{0\}$. Then $T_{x}=T_{y}$ if and only if $\hat{x} \in F \hat{y}$.

This lemma is obvious.

Lemma 4.3. Let $x_{1}, x_{2} \in A_{d} \backslash\{z\}$. If one of the following conditions holds:

(a) $x_{2} \in A_{d}^{+}$and $x_{1} \in A_{d, i}$ for certain $i \in I$,

(b) $x_{1}, x_{2} \in A_{d}^{+}$and $T_{x_{1}-z} \neq T_{x_{2}-z}$,

then

$$
\left[L_{x_{1}}, L_{x_{2}}\right]=L_{x_{1}+x_{2}} .
$$

Proof. Suppose (a) is satisfied. If $d_{i}\left(x_{2}\right)>0$, then $d_{i} \in T_{x_{1}-z} \backslash\left(T_{x_{2}} \cup T_{x_{2}-z}\right)$, hence $F d_{i}+\left\langle d_{i}\left(x_{2}\right) \partial-\partial\left(x_{1}\right) d_{i} \mid \partial \in T_{x_{2}-z}\right\rangle=T$. We deduce that $\left\langle d_{i}\left(x_{2}\right) \partial-\right.$ $\partial\left(x_{1}\right) d_{i}\left|\partial \in T_{x_{2}-z}\right\rangle=T_{x_{1}+x_{2}-z}$. From

$$
\left[t^{x_{1}} d_{i}, t^{x_{2}} \partial\right]=t^{x_{1}+x_{2}}\left(d_{i}\left(x_{2}\right) \partial-\partial\left(x_{1}\right) d_{i}\right), \forall \partial \in T_{x_{2}-z},
$$

we see that (4.2) follows.

If $d_{i}\left(x_{2}\right)=0$, then $x_{1}+x_{2} \in A_{d, i}$. If $x_{1}+x_{2}=z$ further, it follows from $\left(S_{d}\right)_{z}=0$ that (4.2) follows. Suppose $x_{1}+x_{2} \neq z$, by Lemma 4.2 then $T_{x_{2}-z} \neq T_{x_{1}}$. Choose $\partial \in T_{x_{2}-z} \backslash T_{x_{1}}$, so $\left[t^{x_{1}} d_{i}, t^{x_{2}} \partial\right]=-\partial\left(x_{1}\right) t^{x_{1}+x_{2}} d_{i}$. Since $\left(S_{d}\right)_{x_{1}+x_{2}}=F t^{x_{1}+x_{2}} d_{i}$, hence (4.2) follows again.

Suppose (b) is satisfied. We have

$$
\left[t^{x_{1}} \partial_{1}, t^{x_{2}} \partial_{2}\right]=t^{x_{1}+x_{2}}\left(\partial_{1}\left(x_{2}\right) \partial_{2}-\partial_{2}\left(x_{1}\right) \partial_{1}\right)
$$

for all $\partial_{1} \in T_{x_{1}-z}, \partial_{2} \in T_{x_{2}-z}$.

If $x_{1}=0$, it follows from $T_{x_{1}-z} \neq T_{x_{2}-z}$ that $\hat{x}_{2} \notin F \hat{z}$. Then $T_{x_{2}} \neq T_{z}$. Choose $\partial_{1} \in T_{x_{2}} \backslash T_{z}$. Then (4.2) follows from (4.3). Now we assume that $x_{1}, x_{2} \in A_{d}^{+} \backslash\{0\}$.

We claim that $T_{x_{1}-z} \neq T_{x_{2}}$ or $T_{x_{1}} \neq T_{x_{2}-z}$. Otherwise from $T_{x_{1}-z}=T_{x_{2}}$ and $T_{x_{1}}=T_{x_{2}-z}$, by Lemma 4.2 we obtain

$$
d_{1}\left(x_{2}\right)\left(x_{1}-z\right)=\left(d_{1}\left(x_{1}\right)+1\right) x_{2}, d_{1}\left(x_{1}\right)\left(x_{2}-z\right)=\left(d_{1}\left(x_{2}\right)+1\right) x_{1} .
$$

Then $x_{1}=-d_{1}\left(x_{1}\right) z$ and $x_{2}=-d_{1}\left(x_{2}\right) z$, it contradicts $T_{x_{1}-z} \neq T_{x_{2}-z}$. Hence our claim holds.

We assume that $T_{x_{1}-z} \neq T_{x_{2}}$. If $T_{x_{1}-z} \backslash T_{x_{2}} \subset T_{x_{2}-z}$, then we deduce that $T_{x_{1}-z}=T_{x_{2}}$, it is impossible. So $T_{x_{1}-z} \backslash\left(T_{x_{2}} \cup T_{x_{2}-z}\right) \neq \emptyset$. Choose $\partial_{1} \in T_{x_{1}-z} \backslash\left(T_{x_{2}} \cup T_{x_{2}-z}\right)$, then $F \partial_{1}+\left\langle\partial_{1}\left(x_{2}\right) \partial_{2}-\partial_{2}\left(x_{1}\right) \partial_{1} \mid \partial_{2} \in T_{x_{2}-z}\right\rangle=T$. Hence $\left\langle\partial_{1}\left(x_{2}\right) \partial_{2}-\partial_{2}\left(x_{1}\right) \partial_{1} \mid \partial_{2} \in T_{x_{2}-z}\right\rangle=T_{x_{1}+x_{2}-z}$. From (4.3) it follows that (4.2) holds.

Lemma 4.4. For a fixed $i \in I$ and a fixed $x_{0} \in A_{d} \backslash\{z\}$ with $d_{i}\left(x_{0}\right)=1$, the subspace

$$
\left(S_{d}\right)_{x_{0}}+\sum_{x \in A_{d}, d_{i}(x) \leq 0}\left(S_{d}\right)_{x}
$$

generates $S_{d}$ as a Lie algebra. 
Proof. Denote by $M$ the subalgebra generated by the above subspace. We shall show that $\left(S_{d}\right)_{x} \subset M$ for $x \in A_{d}$ by induction on $k=d_{i}(x)$. By definition of $M$ this is true for $x \in A_{d}$ with $d_{i}(x)<1$.

Claim 1. We can assume that $x_{0} \in A_{d}^{0}$.

If $d_{j}\left(x_{0}\right)=-1$ for one $j \in I \backslash\{i\}$, choose $u_{j} \in A_{d, j}^{\#}$, by Lemma 4.3 we deduce that $\left[\left(S_{d}\right)_{x_{0}},\left(S_{d}\right)_{-u_{j}}\right]=\left(S_{d}\right)_{x_{0}-u_{j}} \subset M$. Note that $d_{j}\left(x_{0}-u_{j}\right)=0$. Then we may assume that $x_{0} \in A_{d}^{+}$.

If $d_{j}\left(x_{0}\right)>0$ for some $j \in I \backslash\{i\}$, also using $u_{j} \in A_{d, j}^{\#}$, by Lemma 4.3 we obtain that $\left[\left(S_{d}\right)_{x_{0}},\left(S_{d}\right)_{u_{j}}\right]=\left(S_{d}\right)_{x_{0}+u_{j}} \subset M$. Note that $d_{j}\left(x_{0}+u_{j}\right)=$ $d_{j}\left(x_{0}\right)-1$. Thus after finitely many such steps we may assume that $x_{0} \in A_{d}^{0}$. This is our Claim 1.

Claim 2. There exists $y_{0} \in A_{d}^{0} \backslash\{0\}$ such that $\left(S_{d}\right)_{x_{0}},\left(S_{d}\right)_{x_{0}+y_{0}} \subset M$.

Since $1<|I|<\operatorname{dim} T$ we know that $A_{d}^{0} \neq 0$. Choose $y^{\prime} \in A_{d}^{0} \backslash\{0\}$. If $T_{x_{0}-z}=T_{y^{\prime}-z}$, then $x_{0}-z=2 y^{\prime}-2 z$, i.e., $x_{0}-2 y^{\prime}+z=0$, we set $y_{0}=2 y^{\prime}$. If $T_{x_{0}-z} \neq T_{y^{\prime}-z}$ we set $y_{0}=y^{\prime}$. Thus $y_{0} \in A_{d}^{0} \backslash\{0\}$ and $T_{x_{0}-z} \neq T_{y^{\prime}-z}$. Since $\left(S_{d}\right)_{x_{0}},\left(S_{d}\right)_{y_{0}} \subset M$ and $\left[\left(S_{d}\right)_{x_{0}},\left(S_{d}\right)_{y_{0}}\right]=\left(S_{d}\right)_{x_{0}+y_{0}}$ (Lemma 4.3), then $\left(S_{d}\right)_{x_{0}},\left(S_{d}\right)_{x_{0}+y_{0}} \subset M$. Claim 2 follows.

Suppose that $x \in A_{d}$ with $d_{i}(x)=1$. Note that $T_{x_{0}-z}=T_{x-x_{0}-z}$ implies $x-x_{0}-z=2\left(x_{0}-z\right)$, i.e., $x-3 x_{0}-z=0$, and that $T_{x_{0}+y_{0}-z}=T_{x-x_{0}-y_{0}-z}$ implies $x-3 x_{0}-3 y_{0}+z=0$. Then $T_{x_{0}-z} \neq T_{x-x_{0}-z}$ or $T_{x_{0}+y_{0}-z} \neq$ $T_{x-x_{0}-y_{0}-z}$. Without loss of generality we assume that $T_{x_{0}-z} \neq T_{x-x_{0}-z}$. By Lemma 4.3 we know that $\left[\left(S_{d}\right)_{x-x_{0}},\left(S_{d}\right)_{x_{0}}\right]=\left(S_{d}\right)_{x}$. By noting that $\left(S_{d}\right)_{x-x_{0}},\left(S_{d}\right)_{x_{0}} \subset M$, we get that $\left(S_{d}\right)_{x} \subset M$. So $\left(S_{d}\right)_{x} \subset M$ for $x \in A_{d}$ with $d_{i}(x) \leq 1$.

Suppose that $\left(S_{d}\right)_{x_{0}} \subset M$ for all $x_{0} \in A_{d}$ with $d_{i}\left(x_{0}\right)=k$ for a fixed $k \geq 1$. Consider $x \in A_{d}$ with $d_{i}(x)=k+1$. Similar to the above argument we know that $T_{x_{0}-z} \neq T_{x-x_{0}-z}$ or $T_{x_{0}+y_{0}-z} \neq T_{x-x_{0}-y_{0}-z}$, say $T_{x_{0}-z} \neq T_{x-x_{0}-z}$, By Lemma 4.3 we obtain that $\left[\left(S_{d}\right)_{x-x_{0}},\left(S_{d}\right)_{x_{0}}\right]=\left(S_{d}\right)_{x}$. By noting that $\left(S_{d}\right)_{x-x_{0}},\left(S_{d}\right)_{x_{0}} \subset M$, we get that $\left(S_{d}\right)_{x} \subset M$.

By induction we obtain that $\left(S_{d}\right)_{x} \subset M$ for all $x \in A_{d}$. This completes the proof of Lemma 4.4 .

Lemma 4.5. (a) Suppose that $D_{1}, D_{2}$ are derivations of a Lie algebra $\mathbf{g}$, and that $M \subset \mathbf{g}$ generates $\mathbf{g}$. If $\left.D_{1}\right|_{M}=\left.D_{2}\right|_{M}$, then $D_{1}=D_{2}$.

(b) Suppose that $D_{1}, D_{2} \in \operatorname{Der}\left(S_{d}\right)$ are homogeneous of degree $y \in A_{d}$. If $D_{1}(u)=D_{2}(u)$ for all $u \in\left(S_{d}\right)_{x}$ with $x \in A_{d}^{+}$, i.e., $\left.D_{1}\right|_{S_{d}^{+}}=\left.D_{2}\right|_{S_{d}^{+}}$, then $D_{1}=D_{2}$.

Proof. (a) is obvious. 
(b) It suffices to show that $D_{1}\left(t^{x} d_{1}\right)=D_{2}\left(t^{x} d_{1}\right)$ for $x \in A_{d, 1}$. If $x+y \notin$ $A_{d} \backslash\{z\}$, we see that $D_{1}\left(t^{x} d_{1}\right)=D_{2}\left(t^{x} d_{1}\right)=0$. Suppose that $x+y \in A_{d} \backslash\{z\}$, and that $D_{1}\left(t^{x} d_{1}\right)=t^{x+y} \partial \neq 0$ and $D_{2}\left(t^{x} d_{1}\right)=t^{x+y} \partial^{\prime}$. If $\partial \neq \partial^{\prime}$ we choose $x^{\prime} \in A_{d}^{+}$with $x^{\prime}+x \in A_{d}^{+}$such that $\partial\left(x^{\prime}\right)-\partial^{\prime}\left(x^{\prime}\right) \neq 0$. For any $\partial_{1} \in T_{x^{\prime}-z}$, we have

$$
\left[t^{x^{\prime}} \partial_{1}, t^{x} d_{1}\right]=t^{x^{\prime}+x}\left(\partial_{1}(x) d_{1}-d_{1}\left(x^{\prime}\right) \partial_{1}\right) .
$$

Then $\left[t^{x^{\prime}} \partial_{1}, t^{x+y} \partial\right]=\left[t^{x^{\prime}} \partial_{1}, t^{x+y} \partial^{\prime}\right]$, i.e.,

$$
\partial_{1}(x+y) \partial-\partial\left(x^{\prime}\right) \partial_{1}=\partial_{1}(x+y) \partial^{\prime}-\partial^{\prime}\left(x^{\prime}\right) \partial_{1},
$$

so, we deduce that

$$
\partial_{1}(x+y)\left(\partial-\partial^{\prime}\right)=\left(\partial\left(x^{\prime}\right)-\partial^{\prime}\left(x^{\prime}\right)\right) \partial_{1}, \forall \partial_{1} \in T_{x^{\prime}-z} .
$$

This is impossible since $\operatorname{dim} T_{x^{\prime}-z}>1$. Thus we get a contradiction. Consequently $\partial=\partial^{\prime}$, i.e., $D_{1}\left(t^{x} d_{1}\right)=D_{2}\left(t^{x} d_{1}\right)$.

Now we are ready to describe the homogeneous derivations $D_{y}$ in (4.1).

Proposition 4.6. If $y \notin A_{d}$, then every homogeneous derivation $D$ of $S_{d}$ of degree $y$ is 0 .

Proof. We shall divide the proof into three cases.

Case 1. Suppose $d_{i}(y) \leq-3$ for some $i \in I$.

From Corollary 3.2 we see that $D\left(\left(S_{d}\right)_{x}\right)=0$ for all $x \in A_{d}$ with $d_{i}(x) \leq 1$. By Lemmas 4.4 and 4.5 it follow that $D=0$.

Case 2. Suppose $d_{1}(y)=-2$ and $d_{i}(y) \geq-2$ for all $i \in I$.

Then $D\left(\left(S_{d}\right)_{x}\right)=0$ for $x \in A_{d}$ with $d_{1}(x) \leq 0$. If $|I|=1$, since $\left(S_{d}\right)_{z}=0$ then $D\left(\left(S_{d}\right)_{z-y}\right) \subset\left(S_{d}\right)_{z}=0$ and $d_{1}(z-y)=1$. By Lemma 4.4 it follows that $D=0$.

Suppose $|I|>1$. Assume that $\left\{u_{1}, u_{2}, \cdots, u_{n}\right\} \subset A$ such that $d_{i}\left(u_{j}\right)=$ $-\delta_{i, j}$. If $d_{i}(y) \leq 0$ for some $i \in I \backslash\{1\}$, it follows from $d_{1}\left(u_{i}-u_{1}+y\right)=$ $-1, d_{i}\left(u_{i}-u_{1}+y\right)<0$ that $D\left(\left(S_{d}\right)_{u_{i}-u_{1}}\right) \subset\left(S_{d}\right)_{u_{i}-u_{1}+y}=0$. By Lemmas 4.4 and 4.5 , we also have $D=0$.

Suppose $|I|>1$ and $d_{i}(y)>0$ for all $i \in I \backslash\{1\}$. Choose $v_{1} \in A_{1}^{\#}, v_{2} \in$ $A_{2}^{\#}$. Let $D\left(t^{v_{2}-v_{1}} d_{2}\right)=\lambda t^{v_{2}-v_{1}+y} d_{1}$ for some $\lambda \in F$. From $\left[t^{v_{2}-v_{1}} d_{2}, d_{1}-\right.$ $\left.d_{2}\right]=-2 t^{v_{2}-v_{1}} d_{2}$ and $D\left(d_{1}-d_{2}\right)=0$ we get that $\lambda\left[t^{v_{2}-v_{1}+y} d_{1}, d_{1}-d_{2}\right]=$ $-2 \lambda t^{v_{2}-v_{1}+y} d_{1}$. Then $\lambda\left(d_{1}(y)-d_{2}(y)\right)=0$. Since $d_{1}(y)-d_{2}(y)<-3$ we obtain that $\lambda=0$, i.e., $D\left(\left(S_{d}\right)_{v_{i}-v_{1}}\right)=0$. By Lemmas 4.4 and 4.5 , it follows that $D=0$.

Case 3. Suppose that $|I| \geq 2$, that $d_{i}(y) \geq-1$ for all $i \in I$ and that $d_{1}(y)=d_{2}(y)=-1$. We first show that $D\left(\left(S_{d}\right)_{x}\right)=0$ for all $x \in A_{d}$ with $d_{1}(x) \leq 0$. If $x+y \notin A_{d}$ then $D\left(\left(S_{d}\right)_{x}\right) \subset\left(S_{d}\right)_{x+y}=0$. In particular, $D\left(T_{z}\right)=0$. Suppose $x \in A_{d}$ with $d_{1}(x)=0$ and $x+y \in A_{d}$. then $x+y \in A_{1}$ and $d_{2}(x) \geq 1, d_{i}(x) \geq 0, \forall i>1$. If $y \neq z$ then $\hat{y} \notin F \hat{z}$. Choose $\partial_{1} \in T_{z} \backslash T_{y}$. 
Let $D\left(t^{x} \partial\right)=\lambda t^{x+y} d_{1}$ where $\lambda \in F$. Applying $D$ to $\left[\partial_{1}, t^{x} \partial\right]=\partial_{1}(x) t^{x} \partial$, we obtain that $\lambda \partial_{1}(y)=0$. thus $\lambda=0$. Consequently $D\left(\left(S_{d}\right)_{x}\right)=0$ in this case.

Suppose that $y=z$. Recall that $x, x+y(=x+z) \in A_{d}, d_{1}(x)=0$ and $d_{i}(x)>0$ for all $i \in I \backslash\{1\}$. Choose $u_{j} \in A_{j}^{\#}, \forall j \in I$. For any $x_{0} \in A_{d}^{0} \backslash\{0\}$ we have

$$
\left[t^{x_{0}} \partial, t^{-u_{1}} \partial_{1}\right]=-t^{x_{0}-u_{1}}\left(\partial\left(u_{1}\right) \partial_{1}+\partial_{1}\left(x_{0}\right) \partial\right),
$$

where $\partial \in T_{x_{0}-z}, \partial_{1} \in T_{u_{1}+z}$. Let $D\left(t^{-u_{1}} \partial_{1}\right)=\lambda_{\partial_{1}} t^{z-u_{1}} d_{2}$, where $\lambda_{\partial_{1}} \in F$. Since $\hat{x}_{0}, \hat{z}, \hat{u}_{1}$ are linearly independent, we can choose $\partial \in T_{x_{0}-z} \cap T_{z-u_{1}} \backslash T_{z}$. Then $\left\langle\partial\left(u_{1}\right) \partial_{1}+\partial_{1}\left(x_{0}\right) \partial \mid \partial_{1} \in T_{u_{1}+z}\right\rangle=T_{x_{0}-u_{1}-z}$ since $\partial \notin T_{u_{1}+z}$ and $\left\langle\partial, \partial\left(u_{1}\right) \partial_{1}+\partial_{1}\left(x_{0}\right) \partial \mid \partial_{1} \in T_{u_{1}+z}\right\rangle=T$. Applying $D$ to (4.4) we obtain that

$$
\begin{gathered}
-D\left(t^{x_{0}-u_{1}}\left(\partial\left(u_{1}\right) \partial_{1}+\partial_{1}\left(x_{0}\right) \partial\right)\right)=\left[t^{x_{0}} \partial, \lambda_{\partial_{1}} t^{z-u_{1}} d_{2}\right] \\
=\lambda_{\partial_{1}} \partial\left(z-u_{1}\right) t^{z-u_{1}} d_{2}=0,
\end{gathered}
$$

for all $\partial_{1} \in T_{u_{1}+z}$. Then $D\left(\left(S_{d}\right)_{x_{0}-u_{1}}\right)=0$. Similarly we can get $D\left(\left(S_{d}\right)_{x_{0}-u_{2}}\right)=0$. By $\left[\left(S_{d}\right)_{-x_{0}},\left(S_{d}\right)_{x_{0}-u_{2}}\right]=\left(S_{d}\right)_{-u_{2}}$ we have $D\left(\left(S_{d}\right)_{-u_{2}}\right)=$ 0 . By induction on $k=d_{2}(x) \geq 2$, and using $\left[\left(S_{d}\right)_{-u_{2}},\left(S_{d}\right)_{x+u_{2}}\right]=\left(S_{d}\right)_{x}$, we can obtain that $D\left(\left(S_{d}\right)_{x}\right)=0$ for $x \in A_{d}$ with $d_{1}(x)=0$.

Next we claim that $D\left(\left(S_{d}\right)_{-u_{1}}\right)=0$ for $u_{1} \in A$.

If $y-u_{1} \notin A_{d}$ we have $D\left(\left(S_{d}\right)_{-u_{1}}\right) \subset\left(S_{d}\right)_{y-u_{1}}=0$.

Suppose that $y-u_{1} \in A_{d}$. Then $d_{i}(y) \geq 0$ for all $i \geq 3$. If $y=z$, from the above argument we know that $D\left(\left(S_{d}\right)_{-u_{1}}\right)=0$. Suppose also that $y \neq z$. Choose $\partial_{1} \in T_{z} \backslash T_{y}$. Let $D\left(t^{-u_{1}} \partial\right)=\lambda_{\partial} t^{y-u_{1}} d_{2}$ for $\partial \in$ $T_{z+u_{1}}$, where $\lambda_{\partial} \in F$. Then by $\left[\partial_{1}, t^{-u_{1}} \partial\right]=-\partial_{1}\left(u_{1}\right) t^{-u_{1}} \partial$ we obtain that $\left[\partial_{1}, \lambda_{\partial} t^{y-u_{1}} d_{2}\right]=-\lambda_{\partial} \partial_{1}\left(u_{1}\right) t^{-u_{1}} d_{2}$. Thus $\lambda_{\partial} \partial_{1}(y)=0$. Since $\partial_{1}(y) \neq 0$ we infer that $\lambda_{\partial}=0$. Consequently $D\left(\left(S_{d}\right)_{-u_{1}}\right)=0$ also. Therefore our claim is true. By Lemmas 4.4 and 4.5 we conclude that $D=0$ in this case. Hence we have proved that $D=0$ when $y \notin A_{d}$.

Proposition 4.7. Suppose that $y \in A_{d} \backslash\{0\}$, and that $D \in \operatorname{Der}\left(S_{d}\right)$ is homogeneous of degree $y$.

(a) If $y \neq z$, there exists $t^{y} \partial_{0} \in\left(S_{d}\right)_{y}$ such that $D=\operatorname{ad}\left(t^{y} \partial_{0}\right)$.

(b) If $y=z$, we have $|I|=1$ and $D \in F \cdot \operatorname{ad}\left(t^{y} d_{1}\right)$.

Proof. For any $x \in A_{d} \backslash\{z\}$, we define the linear map $D_{x}: T_{x-z} \rightarrow T_{x+y-z}$ (or $D_{x}: F d_{i} \rightarrow T_{x+y-z}$ if $x \in A_{i}$ ) by $D\left(t^{x} \partial\right)=t^{x+y}\left(D_{x} \partial\right)$. By applying $D$ to

$$
\left[t^{x_{1}} \partial_{1}, t^{x_{2}} \partial_{2}\right]=t^{x_{1}+x_{2}}\left(\partial_{1}\left(x_{2}\right) \partial_{2}-\partial_{2}\left(x_{1}\right) \partial_{1}\right)
$$

where $x_{1}, x_{2} \in A_{d} \backslash\{z\}$ and $\partial_{1} \in T_{x_{1}-z}, \partial_{2} \in T_{x_{2}-z}$, we obtain that

$$
\begin{aligned}
& \left\langle D_{x_{1}} \partial_{1}, x_{2}\right\rangle \partial_{2}-\left\langle D_{x_{2}} \partial_{2}, x_{1}\right\rangle \partial_{1}+\partial_{1}\left(x_{2}+y\right) D_{x_{2}} \partial_{2}-\partial_{2}\left(x_{1}+y\right) D_{x_{1}} \partial_{1} \\
& =D_{x_{1}+x_{2}}\left(\partial_{1}\left(x_{2}\right) \partial_{2}-\partial_{2}\left(x_{1}\right) \partial_{1}\right)
\end{aligned}
$$


holds for $\partial_{1} \in T_{x_{1}-z}, \partial_{2} \in T_{x_{2}-z}$, and $x_{1}, x_{2} \in A_{d} \backslash\{z\}$.

Case 1. Suppose that $\hat{y}$ and $\hat{z}$ are linearly independent. By setting $x_{2}=0$ in (4.5) we obtain

$$
\partial_{1}(y) D_{0}\left(\partial_{2}\right)=\partial_{2}(y) D_{x_{1}}\left(\partial_{1}\right)+\left\langle D_{0}\left(\partial_{2}\right), x_{1}\right\rangle \partial_{1} .
$$

By setting here $x_{2}=0$ in (4.6) we obtain that

$$
\partial_{1}(y) D_{0}\left(\partial_{2}\right)=\partial_{2}(y) D_{0}\left(\partial_{1}\right) .
$$

Choose $\partial_{2} \in T_{z} \backslash T_{y}$, and denote $\partial_{0}=\partial_{2}(y)^{-1} D_{0}\left(\partial_{2}\right)$. Then we have $\partial_{0} \in$ $T_{y-z}$ and

$$
D_{0}\left(\partial_{1}\right)=-\partial_{1}(y) \partial_{0}, \forall \partial_{1} \in T_{z} .
$$

Hence we can rewrite (4.6) as

$$
\partial_{2}(y)\left(D_{x_{1}}\left(\partial_{1}\right)-\partial_{1}(y) \partial_{0}+\partial_{0}\left(x_{1}\right) \partial_{1}\right)=0 .
$$

Thus we deduce that

$$
D_{x_{1}}\left(\partial_{1}\right)=\partial_{1}(y) \partial_{0}-\partial_{0}\left(x_{1}\right) \partial_{1} .
$$

It follows that $D=-$ ad $\left(t^{y} \partial_{0}\right)$. Note that by now we have not known that $t^{y} \partial_{0} \in S_{d}$ yet. If $\partial_{0}=0$ or $y \in A_{d}^{+}$, from $\partial_{0} \in T_{y-z}$ then $t^{y} \partial_{0} \in S_{d}$. If $\partial_{0} \neq 0$ and $y \in A_{i}$ for some $i \in I$, since $A_{i} \not \subset \operatorname{ker}\left(\partial_{0}\right)$, choose $x_{0} \in A_{i} \backslash \operatorname{ker}\left(\partial_{0}\right)$. Since $D\left(\left(S_{d}\right)_{x_{0}}\right)=0$ and $D=$ ad $\left(t^{y} \partial_{0}\right)$, we deduce that

$$
\left[t^{y} \partial_{0}, t^{x_{0}} d_{i}\right]=t^{x_{0}+y}\left(\partial_{0}\left(x_{0}\right) d_{i}+\partial_{0}\right)=0 .
$$

Thus $\partial_{0}=-\partial_{0}\left(x_{0}\right) d_{i}$. Hence $t^{y} \partial_{0} \in S_{d}$.

Case 2. Suppose that $y=z$. Then $|I|=1$ and $z \in A_{1}^{\#}$. Since $D_{0}\left(\left(S_{d}\right)_{0}\right) \subset$ $\left(S_{d}\right)_{z}=0$, we know that $D_{0}=0$.

Claim 1. For $x_{1} \in A_{d} \backslash\{z\}$ with $\hat{x}_{1} \notin F \hat{z}$, there exists a constant $a_{x_{1}} \in F$ such that

$$
D_{x_{1}} \partial=a_{x_{1}} \partial, \forall \partial \in T_{x_{1}} \cap T_{z} .
$$

If $x_{1} \in A_{d} \backslash A_{d}^{+}$, clearly (4.8) is true. Next we suppose that $x_{1} \in A_{d}^{+}$. By setting $\partial_{1}=\partial_{2}=\partial \in T_{x_{1}} \cap T_{z}$ and $x_{2}=-z$ in (4.5), we obtain that

$$
-\left\langle D_{x_{1}} \partial, z\right\rangle \partial-\left\langle D_{-z} \partial, x_{1}\right\rangle \partial=0
$$

and so

$$
\left\langle D_{-z} \partial, x_{1}\right\rangle=-\left\langle D_{x_{1}} \partial, z\right\rangle
$$

holds. On the other hand, for $x_{2}=-z, \partial_{2}=\partial \in T_{x_{1}} \cap T_{z}$, and arbitrary $\partial_{1} \in T_{x_{1}-z}$, (note that we allow $x_{1} \in A_{1}$ here), (4.5) gives that

$$
\left\langle D_{x_{1}} \partial_{1}, z\right\rangle \partial+\left\langle D_{-z} \partial, x_{1}\right\rangle \partial_{1}=\partial_{1}(z) D_{x_{1}-z} \partial .
$$


By evaluating both sides at $x_{1}$ and using $\partial_{1}\left(x_{1}\right)=\partial_{1}(z)$, we obtain that

$$
\partial_{1}(z)\left[\left\langle D_{x_{1}-z} \partial, x_{1}\right\rangle-\left\langle D_{-z} \partial, x_{1}\right\rangle\right]=0 .
$$

As $\hat{x}_{1} \notin F \hat{z}$, we can choose $\partial_{1} \in T_{x_{1}-z} \backslash T_{z}$, and so

$$
\left\langle D_{x_{1}-z} \partial, x_{1}\right\rangle=\left\langle D_{-z} \partial, x_{1}\right\rangle, \forall \partial \in T_{x_{1}} \cap T_{z} .
$$

By substituting $x_{1}+z$ for $x_{1}$ in (4.9), and using $\left\langle D_{-z} \partial, z\right\rangle=0$, we infer that

$$
-\left\langle D_{x_{1}-z} \partial, x_{1}\right\rangle=\left\langle D_{-z} \partial, x_{1}\right\rangle
$$

holds for $\partial \in T_{x_{1}} \cap T_{z}$. By comparing this equation with (4.11), we conclude that

$$
\left\langle D_{-z} \partial, x_{1}\right\rangle=0, \forall x_{1} \in A_{d}, \partial \in T_{x_{1}} \cap T_{z} .
$$

Now (4.10) gives that

$$
\partial_{1}(z) D_{x_{1}-z} \partial=\left\langle D_{x_{1}} \partial_{1}, z\right\rangle \partial
$$

for $\partial_{1} \in T_{x_{1}-z}, \partial \in T_{x_{1}} \cap T_{z}, x_{1} \in A_{d}$, with $\hat{x}_{1} \notin F \hat{z}$. By choosing $\partial_{1} \in$ $T_{x_{1}-z} \backslash T_{z}$ and setting $a_{x_{1}-z}=\frac{\left\langle D_{x_{1}} \partial_{1}, z\right\rangle}{\partial_{1}(z)}$, then we have $D_{x_{1}-z} \partial=a_{x_{1}-z} \partial$, thus

$$
D_{x_{1}} \partial=a_{x_{1}} \partial, \forall x_{1} \in A_{d}, \partial \in T_{x_{1}} \cap T_{z} .
$$

Hence our first claim is proved.

Claim 2. If $x_{1}, x_{2} \in A_{d}^{+}$with $\hat{x}_{1}, \hat{x}_{2}, \hat{x}_{1}+\hat{x}_{2} \notin F \hat{z}$, then

$$
a_{x_{1}+x_{2}}=a_{x_{1}}+a_{x_{2}} \text {. }
$$

In order to prove this claim we shall consider first the case where $\hat{x}_{1}, \hat{x}_{2}$, and $\hat{z}$ are linearly independent. Then we can choose $\partial_{1} \in\left(T_{x_{1}} \cap T_{z}\right) \backslash T_{x_{2}}$ and $\partial_{2} \in\left(T_{x_{2}} \cap T_{z}\right) \backslash T_{x_{1}}$. It follows that $\partial_{1}\left(x_{2}\right) \partial_{2}-\partial_{2}\left(x_{1}\right) \partial_{1}$ is a nonzero vector in $T_{x_{1}+x_{2}} \cap T_{z}$. By using the first claim, (4.5) gives that

$$
\left(a_{x_{1}+x_{2}}-a_{x_{1}}-a_{x_{2}}\right)\left[\partial_{1}\left(x_{2}\right) \partial_{2}-\partial_{2}\left(x_{1}\right) \partial_{1}\right]=0,
$$

and so (4.13) holds in this case.

Now assume that $\hat{x}_{1}, \hat{x}_{2}$ and $\hat{z}$ are linearly dependent. Since $\operatorname{dim} T \geq 3$ we can choose $w \in A$ such that $\hat{x}_{1}, \hat{w}$ and $\hat{z}$ are linearly independent. By using the case already established, we have

$$
a_{x_{1}}=a_{x_{1}+w}+a_{-w}=a_{x_{1}}+a_{w}+a_{-w},
$$

and

$$
a_{x_{1}+x_{2}}=a_{x_{1}+w}+a_{x_{2}-w}=a_{x_{1}}+a_{w}+a_{x_{2}}+a_{-w},
$$

and conclude again that (4.13) holds. Hence our second claim is proved.

Now let $x \in A_{d}^{+}$with $\hat{x} \in F \hat{z}$ and set $a_{x}=a_{x+v}-a_{v}$ where $v \in A_{d}^{+}$with $\hat{v} \notin F \hat{z}$. By our second claim, $a_{x+v}-a_{v}$ is independent of the choice of $v$. 
With $a_{x}$ now defined for all $x \in A_{d}^{+}$, it is easy to see that (4.13) is valid for all $x_{1}, x_{2} \in A_{d}^{+}$.

We shall now remove the restriction $\hat{x}_{1} \notin F \hat{z}$ in (4.8). Thus assume that $\hat{x}_{1} \in F \hat{z}$ and $x_{1} \in A_{d}^{+}$. We choose $x_{2} \in A_{d}^{+}$so that $\hat{x}_{2} \notin F \hat{z}$, and let $\partial_{1} \in T_{z}$ and $\partial_{2} \in T_{x_{2}} \cap T_{z}$. By using the first claim and $\partial_{2}\left(x_{1}\right)=$ $\partial_{1}(y)=0$, the equation (4.5) gives $\left\langle D_{x_{1}} \partial_{1}, x_{2}\right\rangle \partial_{2}=a_{x_{1}} \partial_{1}\left(x_{2}\right) \partial_{2}$. Hence $\left\langle\left(D_{x_{1}}-a_{x_{1}}\right) \partial_{1}, x_{2}\right\rangle=0$ for all $x_{2} \in A_{d}^{+}$with $\hat{x}_{2} \notin F \hat{z}$, and so (4.8) holds also for $\hat{x}_{1} \in F \hat{z}$.

For $x \in A_{d}^{+}$, we define linear maps $D_{x}^{\prime}: T_{x-z} \rightarrow T$ by $D_{x}^{\prime} \partial=D_{x} \partial-a_{x} \partial$. By Claim 1, $T_{x} \cap T_{z}$ is contained in the kernel of $D_{x}^{\prime}$. In particular, if $\hat{x} \in F \hat{z}$ and $x \neq z$, then $D_{x}^{\prime}=0$. If $\hat{x} \notin F \hat{z}$, then $T_{x} \cap T_{z}$ is a hyperplane in $T_{x-z}$, and so the vector

$$
\partial_{x}:=\frac{D_{x}^{\prime} \partial}{\partial(y)}
$$

is independent of the choice of $\partial \in T_{x-z} \backslash T_{z}$ (note that $y=z$ ). Thus we have

$$
D_{x}^{\prime} \partial=\partial(y) \partial_{x}, \forall x \in A_{d}^{+}, \partial \in T_{x-z} .
$$

If $\hat{x} \in F \hat{z}$, then $\partial_{x}$ is not defined but (4.15) is also valid because $D_{x}^{\prime}=0$ and $\partial(y)=0$ for $\partial \in T_{x-z}=T_{z}$.

By substituting $D_{x_{1}}^{\prime}+a_{x_{1}}$ for $D_{x_{1}}$ and making similar substitutions for $D_{x_{2}}$ and $D_{x_{1}+x_{2}}$ in (4.5), we obtain that

$$
\begin{aligned}
& \left\langle D_{x_{1}}^{\prime} \partial_{1}, x_{2}\right\rangle \partial_{2}-\left\langle D_{x_{2}}^{\prime} \partial_{2}, x_{1}\right\rangle \partial_{1}+\partial_{1}\left(x_{2}+y\right) D_{x_{2}}^{\prime} \partial_{2} \\
& \quad-\partial_{2}\left(x_{1}+y\right) D_{x_{1}}^{\prime} \partial_{1}+a_{x_{2}} \partial_{1}(x) \partial_{2}-a_{x_{1}} \partial_{2}(x) \partial_{1} \\
& =D_{x_{1}+x_{2}}^{\prime}\left(\partial_{1}\left(x_{2}\right) \partial_{2}-\partial_{2}\left(x_{1}\right) \partial_{1}\right)
\end{aligned}
$$

holds for $x_{1}, x_{2} \in A_{d}^{+}, \partial_{1} \in T_{x_{1}-z}$ and $\partial_{2} \in T_{x_{2}-z}$.

By using (4.15) and similar expressions for $D_{x_{2}}^{\prime}$ and $D_{x_{1}+x_{2}}^{\prime}$, the last equation can be rewritten as follows

$$
\begin{aligned}
& \partial_{1}(y)\left[a_{x_{2}}+\partial_{x_{1}}\left(x_{2}\right)\right] \partial_{2}-\partial_{2}(y)\left[a_{x_{1}}+\partial_{x_{2}}\left(x_{1}\right)\right] \partial_{1} \\
& =\left[\partial_{1}\left(x_{2}\right) \partial_{2}(y)-\partial_{2}\left(x_{1}\right) \partial_{1}(y)\right] \partial_{x_{1}+x_{2}} \\
& \quad+\partial_{1}(y) \partial_{2}\left(x_{1}+y\right) \partial_{x_{1}}-\partial_{2}(y) \partial_{1}\left(x_{2}+y\right) \partial_{x_{2}} .
\end{aligned}
$$

Claim 3. The vector $\partial_{x}$ for $x \in A_{d}^{+}$with $\hat{x} \notin F \hat{z}$ are independent of $x$.

Suppose $x_{1}, x_{2} \in A_{d}^{+}$with $\hat{x}_{1}, \hat{x}_{2}, \hat{x}_{1}+\hat{x}_{2} \notin F \hat{z}$. For $\partial_{1} \in T_{x_{1}} \cap T_{z} \backslash\{0\}$ and $\partial_{2} \in T_{x_{2}-z} \backslash T_{z}$, (4.16) gives

$$
\partial_{1}\left(x_{2}\right)\left(\partial_{x_{2}}-\partial_{x_{1}+x_{2}}\right)=\left[a_{x_{1}}+\partial_{x_{2}}\left(x_{1}\right)\right] \partial_{1} .
$$


If $\hat{x}_{1}, \hat{x}_{2}, \hat{z}$ are linearly dependent, we see that $\partial_{1}\left(x_{2}\right)=0$. Thus we obtain from (4.17) that $a_{x_{1}}=-\partial_{x_{2}}\left(x_{1}\right)$.

Assume that $\hat{x}_{1}, \hat{x}_{2}, \hat{z}$ are linearly independent. Then $\partial_{1} \in T_{x_{1}} \cap T_{z}$ can be chosen so that $\partial_{1}\left(x_{2}\right) \neq 0$. By evaluating both sides of (4.17) at $x_{1}$, we obtain that $\partial_{1}\left(x_{2}\right)\left(\partial_{x_{2}}\left(x_{1}\right)-\partial_{x_{1}+x_{2}}\left(x_{1}\right)\right)=0$. Since $\partial_{1}\left(x_{2}\right) \neq 0$ we deduce that

$$
\partial_{x_{2}}\left(x_{1}\right)=\partial_{x_{1}+x_{2}}\left(x_{1}\right), \forall x_{1}, x_{2} \in A_{d}^{+} \text {, with } \hat{x}_{1}, \hat{x}_{2}, \hat{x}_{1}+\hat{x}_{2} \notin F \hat{z} .
$$

Symmetrically we have

$$
\partial_{x_{1}}\left(x_{2}\right)=\partial_{x_{1}+x_{2}}\left(x_{2}\right), \forall x_{1}, x_{2} \in A_{d}^{+}, \text {with } \hat{x}_{1}, \hat{x}_{2}, \hat{x}_{1}+\hat{x}_{2} \notin F \hat{z} .
$$

By evaluating (4.17) at $x_{2}$ we get

$$
a_{x_{1}}=\partial_{x_{2}}\left(x_{2}-x_{1}\right)-\partial_{x_{1}+x_{2}}\left(x_{2}\right),
$$

i.e.,

$$
a_{x_{1}}=\partial_{x_{2}}\left(x_{2}-x_{1}\right)-\partial_{x_{1}}\left(x_{2}\right) .
$$

By evaluating (4.17) at $z$, we find

$$
\partial_{x_{1}+x_{2}}(z)=\partial_{x_{2}}(z)=\partial_{x_{1}}(z) .
$$

By evaluating (4.16) at $z$, we find that

$$
\partial_{1}(z) \partial_{2}(z)\left[\partial_{x_{1}}\left(x_{2}\right)-\partial_{x_{2}}\left(x_{1}\right)+a_{x_{2}}-a_{x_{1}}\right]=0 .
$$

Since we can choose $\partial_{1} \in T_{x_{1}-z}$ and $\partial_{2} \in T_{x_{2}-z}$ such that $\partial_{1}(z) \partial_{2}(z) \neq 0$, we infer that

$$
a_{x_{1}}-a_{x_{2}}=\partial_{x_{1}}\left(x_{2}\right)-\partial_{x_{2}}\left(x_{1}\right) .
$$

By replacing $x_{1}$ with $x_{1}+x_{2}$ and using Claim 2, we obtain the equation

$$
a_{x_{1}}=\partial_{x_{1}+x_{2}}\left(x_{2}\right)-\partial_{x_{2}}\left(x_{1}+x_{2}\right)=\partial_{x_{1}}\left(x_{2}\right)-\partial_{x_{2}}\left(x_{1}+x_{2}\right) .
$$

Combining this with (4.19), we deduce that $\partial_{x_{1}}\left(x_{2}\right)=\partial_{x_{2}}\left(x_{2}\right)$. Considering (4.19) also we have $a_{x_{1}}=-\partial_{x_{2}}\left(x_{1}\right)$. So $\partial_{x_{1}}\left(x_{2}\right)$ is independent of $x_{1}$, consequently $\partial_{x_{1}}$ is independent of $x_{1}$. Then Claim 3 is proved.

Denote $-\partial_{x}\left(x \in A_{d}^{+}\right.$with $\left.\hat{x} \notin F \hat{z}\right)$ by $\partial_{0}$. So we have

$$
a_{x}=\partial_{0}(x), \forall x \in A_{d}^{+} \text {, with } \hat{x} \notin F \hat{z} .
$$

By the definition of $a_{x_{1}}$ for $x \in A_{d}^{+}$with $\hat{x} \notin F \hat{z}$, we also have

$$
a_{x}=\partial_{0}(x), \forall x \in A_{d}^{+} \text {. }
$$

Combining this with (4.15), we deduce that

$$
D_{x} \partial=-\partial(y) \partial_{0}+\partial_{0}(x) \partial, \forall x \in A_{d}^{+}, \partial \in T_{x-z} .
$$

So $\left.D\right|_{S_{d}^{+}}=\left.\operatorname{ad}\left(t^{y} \partial_{0}\right)\right|_{S_{d}^{+}}$. By Lemma 4.5 we see that $D=\operatorname{ad}\left(t^{y} \partial_{0}\right)$. 
Choose $x \in A_{d}^{0} \backslash\{0\}$, and $\partial \in T_{x-z} \backslash T_{z}$. We see that $D\left(\left(S_{d}\right)_{x}\right) \subset\left(S_{d}\right)_{x+z}=$ $F t^{x+z} d_{1}$, i.e., $D_{x} \partial=\lambda_{\partial} d_{1}$ for some $\lambda_{\partial} \in F$. Combining this with (4.21), we infer that

$$
\lambda_{\partial} d_{1}=-\partial(y) \partial_{0}+\partial_{0}(x) \partial .
$$

Since $d_{1} \notin T_{x-z}$ and $\operatorname{dim} T_{x-z} \geq 2$ we conclude from the above equation that $\partial_{0}(x)=0$. Furthermore we deduce that $\partial_{0}=\lambda_{\partial} \partial(y)^{-1} d_{1}$, i.e., $\partial_{0} \in F d_{1}$.

Case 3. Suppose that $y=-\lambda z$, where $\lambda \in \mathbf{N}=\{1,2,3, \ldots\}$.

By setting $x_{2}=0$ in (4.5) we obtain

$$
\partial_{1}(y) D_{0}\left(\partial_{2}\right)=\left\langle D_{0}\left(\partial_{2}\right), x_{1}\right\rangle \partial_{1},
$$

for $\partial_{1} \in T_{x_{1}-z}, \partial_{2} \in T_{z}$. Since $\operatorname{dim} T_{x_{1}-z} \geq 2$ we deduce that $\left\langle D_{0}\left(\partial_{2}\right), x_{1}\right\rangle=$ 0 for all $x \in A_{d}$. It follows that $D_{0}\left(\partial_{2}\right)=0$ for $\partial_{2} \in T_{z}$, i.e., $D_{0}=0$.

Now we show that Claim 1 is also true in this case.

First suppose that $x_{1} \in A_{d}^{+}$with $-x_{1} \in A_{d}$ and $\hat{x} \notin F \hat{z}$. Since $\hat{x} \notin F \hat{z}$ and $-x_{1}-z \in A_{d}^{+}$, we can choose $\partial_{2} \in T_{-x_{1}-z} \backslash T_{z}$. By setting $x_{2}=-x_{1}$ and $\partial_{1}=\partial \in T_{x_{1}} \cap T_{z}$ in (4.5) and using $D_{0}=0$ we obtain that

$$
\partial_{2}\left(x_{1}+y\right) D_{x_{1}}(\partial)+\left\langle D_{x_{1}}(\partial), x_{1}\right\rangle \partial_{2}+\left\langle D_{-x_{1}}\left(\partial_{2}\right), x_{1}\right\rangle \partial=0 .
$$

By evaluating the above equation at $x_{1}+y-z$ and by using $\left\langle D_{x_{1}}(\partial), x_{1}+\right.$ $y-z\rangle=0, \partial\left(x_{1}\right)=\partial(y)=\partial(z)=0$, we conclude that $\left\langle D_{x_{1}}(\partial), x_{1}\right\rangle=0$, and consequently

$$
\partial_{2}\left(x_{1}+y\right) D_{x_{1}}(\partial)=-\left\langle D_{-x_{1}}\left(\partial_{2}\right), x_{1}\right\rangle \partial
$$

holds for all $\partial \in T_{x_{1}} \cap T_{z}$ and $\partial_{2} \in T_{-x_{1}-z}$. Since $\partial_{2}\left(x_{1}+y\right)=-(\lambda+1) \partial_{2}(z) \neq$ 0 , then (4.8) holds for $x_{1} \in A_{d}^{+}$with $-x_{1} \in A_{d}$ and $\hat{x} \notin F \hat{z}$.

We shall show (4.8) for $x_{1} \in A_{d}^{+}$by induction on $d\left(x_{1}\right):=\sum_{i \in I} d_{i}\left(x_{1}\right)$.

This has been proved for all $x_{1}$ with $d\left(x_{1}\right) \leq 1$. Now suppose (4.8) holds for all $x_{1}$ with $d\left(x_{1}\right) \leq k(\geq 1)$. Consider $x_{2} \in A_{d}^{+}$with $d\left(x_{2}\right)=$ $k+1$. Choose $x_{0} \in A_{d}^{+}$with $d\left(x_{0}\right)=1$ and with $\hat{x}_{0}, \hat{x}_{2}, \hat{z}$ being linearly independent. By inductive hypothesis we have

$$
\begin{gathered}
D_{x_{0}} \partial^{\prime}=a_{x_{0}} \partial^{\prime}, \forall \partial^{\prime} \in T_{x_{0}} \cap T_{z}, \\
D_{x_{2}-x_{0}} \partial=a_{x_{2}-x_{0}} \partial, \forall \partial \in T_{x_{2}-x_{0}} \cap T_{z} .
\end{gathered}
$$

By replacing $x_{1}$ with $x_{0}, x_{2}$ with $x_{2}-x_{0}, \partial_{1}$ with $\partial_{0}^{\prime}, \partial_{2}$ with $\partial$ respectively in (4.5), we obtain that

$$
D_{x_{2}}\left(\partial_{0}^{\prime}\left(x_{2}\right) \partial-\partial\left(x_{0}\right) \partial_{0}^{\prime}\right)=\left(a_{x_{0}}+a_{x_{2}-x_{0}}\right)\left(\partial_{0}^{\prime}\left(x_{2}\right) \partial-\partial\left(x_{0}\right) \partial_{0}^{\prime}\right)
$$

for all $\partial_{0}^{\prime} \in T_{x_{0}} \cap T_{z}, \partial \in T_{x_{2}-x_{0}} \cap T_{z}$. It suffices to show that

$$
\left\langle\partial_{0}^{\prime}\left(x_{2}\right) \partial-\partial\left(x_{0}\right) \partial_{0}^{\prime} \mid \partial_{0}^{\prime} \in T_{x_{0}} \cap T_{z}, \partial \in T_{x_{2}-x_{0}} \cap T_{z}\right\rangle=T_{x_{2}} \cap T_{z} .
$$

Choose $\partial_{0}^{\prime} \in\left(T_{x_{0}} \cap T_{z}\right) \backslash T_{x_{2}}$, then

$$
F \partial_{0}^{\prime}+\left\langle\partial_{0}^{\prime}\left(x_{2}\right) \partial-\partial\left(x_{0}\right) \partial_{0}^{\prime} \mid \partial \in T_{x_{2}-x_{0}} \cap T_{z}\right\rangle=F \partial_{0}^{\prime}+T_{x_{2}-x_{0}} \cap T_{z}
$$


is codimension 1 in $T$. Hence the subspace

$$
\left\langle\partial_{0}^{\prime}\left(x_{2}\right) \partial-\partial\left(x_{0}\right) \partial_{0}^{\prime} \mid \partial \in T_{x_{2}-x_{0}} \cap T_{z}\right\rangle \subset T_{x_{2}} \cap T_{z}
$$

is codimension 2 in $T$. Therefore (4.22) holds. Thus there exists $a_{x_{2}} \in F$ such that $D_{x_{2}} \partial=a_{x_{2}} \partial$ for all $\partial \in T_{x_{2}} \cap T_{z}$. Consequently Claim 1 is true.

Exactly the same as that in Case 2, Claim 2 is true in this case also.

Now same as in Case 2, we define $a_{x}$ for $x \in A_{d}^{+}$with $\hat{x} \in F \hat{z}$ and we can remove the restriction $\hat{x} \notin F \hat{z}$ in (4.8), then we can also define the linear map $D_{x}^{\prime}$, the vector $\partial_{x}$ in (4.14) for $x \in A_{d}^{+}$with $\hat{x} \notin F \hat{z}$, and same as before we also get equations (4.15), (4.16).

Now we claim that Claim 3 is true in this case also. The proof is exactly the same as it was in Case 2.

We also denote $-\partial_{x}\left(x \in A_{d}^{+}\right.$with $\left.\hat{x} \notin F \hat{z}\right)$ by $\partial_{0}$. So we have

$$
a_{x}=\partial_{0}(x), \forall x \in A_{d}^{+} \text {, with } \hat{x} \notin F \hat{z} .
$$

The same as in Case 2, Equations (4.20) and (4.21) are true. So $\left.D\right|_{S_{d}^{+}}=$ ad $\left.\left(t^{y} \partial_{0}\right)\right|_{S_{d}^{+}}$. By Lemma 4.5 we see that $D=\operatorname{ad}\left(t^{y} \partial_{0}\right)$. By definition of $\partial_{0}$ we know that $t^{y} \partial_{0} \in\left(S_{d}\right)_{y}$.

By now we have completed the proof of Proposition 4.7.

Proposition 4.8. Suppose that $D \in \operatorname{Der}\left(S_{d}\right)$ is homogeneous of degree 0 . Then there exists a $\nu \in \operatorname{hom}(A, F)$ such that $D=D_{\nu}$.

Proof. For any $x \in A_{d}^{+}$, we define the linear map $D_{x}: T_{x-z} \rightarrow T_{x-z}$ (or $D_{x}: F d_{i} \rightarrow F d_{i}$ if $\left.x \in A_{i}\right)$ by $D\left(t^{x} \partial\right)=t^{x+y}\left(D_{x} \partial\right)$. If $\partial \in T_{x_{1}-z}$, then $\partial\left(x_{1}\right)=\partial(z)$ and $\left\langle D_{x_{1}} \partial, x_{1}\right\rangle=\left\langle D_{x_{1}} \partial, z\right\rangle$. As $x=0$, the equation (4.5) takes the form

$$
\begin{aligned}
& \left\langle D_{x_{1}} \partial_{1}, x_{2}\right\rangle \partial_{2}-\left\langle D_{x_{2}} \partial_{2}, x_{1}\right\rangle \partial_{1}+\partial_{1}\left(x_{2}\right) D_{x_{2}} \partial_{2}-\partial_{2}\left(x_{1}\right) D_{x_{1}} \partial_{1} \\
& =D_{x_{1}+x_{2}}\left(\partial_{1}\left(x_{2}\right) \partial_{2}-\partial_{2}\left(x_{1}\right) \partial_{1}\right)
\end{aligned}
$$

where $\partial_{1} \in T_{x_{1}-z}, \partial_{2} \in T_{x_{2}-z}$, and $x_{1}, x_{2} \in A_{d}^{+}$. By setting $v=0$ in (4.23), we obtain that $\left\langle D_{0} \partial_{2}, x_{1}\right\rangle \partial_{1}=0$. Hence $D_{0}=0$.

We claim that (4.8) holds for $x_{1} \in A_{d} \backslash\{z\}$ and $\partial \in T_{x_{1}} \cap T_{z}$.

If $x_{1} \in A_{d} \backslash A_{d}^{+}$, (4.8) holds clearly. Next suppose that $x \in A_{d}^{+}$.

First suppose that $x_{1} \in A_{d}^{+}$with $-x_{1} \in A_{d}$ and $\hat{x} \notin F \hat{z}$. Since $\hat{x} \notin F \hat{z}$ and $-x_{1}-z \in A_{d}^{+}$, we can choose $\partial_{2} \in T_{-x_{1}-z} \backslash T_{z}$. By setting $x_{2}=-x_{1}$ and $\partial_{1}=\partial \in T_{x_{1}} \cap T_{z}$ in (4.23) and using $D_{0}=0$, we obtain that

$$
\partial_{2}\left(x_{1}\right) D_{x_{1}}(\partial)+\left\langle D_{x_{1}}(\partial), x_{1}\right\rangle \partial_{2}+\left\langle D_{-x_{1}}\left(\partial_{2}\right), x_{1}\right\rangle \partial=0 .
$$


By evaluating the above equation at $x_{1}-z$ and by using $\left\langle D_{x_{1}}(\partial), x_{1}-z\right\rangle=0$, $\partial\left(x_{1}\right)=\partial(z)=0$, we conclude that $\left\langle D_{x_{1}}(\partial), x_{1}\right\rangle=0$, and consequently

$$
\partial_{2}\left(x_{1}\right) D_{x_{1}}(\partial)=-\left\langle D_{-x_{1}}\left(\partial_{2}\right), x_{1}\right\rangle \partial
$$

holds for all $\partial \in T_{x_{1}} \cap T_{z}$ and $\partial_{2} \in T_{-x_{1}-z}$. Since $\partial_{2}\left(x_{1}\right)=-\partial_{2}(z) \neq 0$, then (4.8) holds for $x_{1} \in A_{d}^{+}$with $-x_{1} \in A_{d}$ and $\hat{x} \notin F \hat{z}$.

We shall show (4.8) for $x_{1} \in A_{d}^{+}$by induction on $d\left(x_{1}\right):=\sum_{i \in I} d_{i}\left(x_{1}\right)$.

This has been proved for all $x_{1}$ with $d\left(x_{1}\right) \leq 1$. Now suppose (4.8) holds for all $x_{1}$ with $d\left(x_{1}\right) \leq k(\geq 1)$. Consider $x_{2} \in A_{d}^{+}$with $d\left(x_{2}\right)=$ $k+1$. Choose $x_{0} \in A_{d}^{+}$with $d\left(x_{0}\right)=1$ and with $\hat{x}_{0}, \hat{x}_{2}, \hat{z}$ being linearly independent. By inductive hypothesis we have

$$
\begin{gathered}
D_{x_{0}} \partial^{\prime}=a_{x_{0}} \partial^{\prime}, \forall \partial^{\prime} \in T_{x_{0}} \cap T_{z}, \\
D_{x_{2}-x_{0}} \partial=a_{x_{2}-x_{0}} \partial, \forall \partial \in T_{x_{2}-x_{0}} \cap T_{z} .
\end{gathered}
$$

By replacing $x_{1}$ with $x_{0}, x_{2}$ with $x_{2}-x_{0}, \partial_{1}$ with $\partial_{0}^{\prime}, \partial_{2}$ with $\partial$ respectively in (4.23), we obtain that

$$
D_{x_{2}}\left(\partial_{0}^{\prime}\left(x_{2}\right) \partial-\partial\left(x_{0}\right) \partial_{0}^{\prime}\right)=\left(a_{x_{0}}+a_{x_{2}-x_{0}}\right)\left(\partial_{0}^{\prime}\left(x_{2}\right) \partial-\partial\left(x_{0}\right) \partial_{0}^{\prime}\right)
$$

for all $\partial_{0}^{\prime} \in T_{x_{0}} \cap T_{z}, \partial \in T_{x_{2}-x_{0}} \cap T_{z}$. It suffices to show that

$$
\left\langle\partial_{0}^{\prime}\left(x_{2}\right) \partial-\partial\left(x_{0}\right) \partial_{0}^{\prime} \mid \partial_{0}^{\prime} \in T_{x_{0}} \cap T_{z}, \partial \in T_{x_{2}-x_{0}} \cap T_{z}\right\rangle=T_{x_{2}} \cap T_{z} .
$$

Choose $\partial_{0}^{\prime} \in\left(T_{x_{0}} \cap T_{z}\right) \backslash T_{x_{2}}$, then

$$
F \partial_{0}^{\prime}+\left\langle\partial_{0}^{\prime}\left(x_{2}\right) \partial-\partial\left(x_{0}\right) \partial_{0}^{\prime} \mid \partial \in T_{x_{2}-x_{0}} \cap T_{z}\right\rangle=F \partial_{0}^{\prime}+T_{x_{2}} \cap T_{z}
$$

is codimension 1 in $T$. Hence the subspace

$$
\left\langle\partial_{0}^{\prime}\left(x_{2}\right) \partial-\partial\left(x_{0}\right) \partial_{0}^{\prime} \mid \partial \in T_{x_{2}-x_{0}} \cap T_{z}\right\rangle \subset T_{x_{2}} \cap T_{z}
$$

is codimension 2 in $T$. Therefore (4.23) holds. Thus there exists $a_{x_{2}} \in F$ such that $D_{x_{2}} \partial=a_{x_{2}} \partial$ for all $\partial \in T_{x_{2}} \cap T_{z}$. Consequently our claim about (4.8) is true.

Exactly the same as that in Case 2 in the proof of Proposition 4.7, Claim 2 in the proof of Proposition 4.7 is true in this case also.

Now as in Case 2 of the proof of Proposition 4.7, we define $a_{x}$ for $x \in$ $A_{d} \backslash\{z\}$ with $\hat{x} \in F \hat{z}$ and we can remove the restriction $\hat{x} \notin F \hat{z}$ in (4.8). Then we have obtained that:

(a) For any $x \in A_{d} \backslash\{z\}$, there exists a constant $a_{x} \in F$ such that

$$
D_{x} \partial=a_{x} \partial, \forall \partial \in T_{x} \cap T_{z} .
$$

(b) For all $x_{1}, x_{2} \in A_{d}^{+}$,

$$
a_{x_{1}+x_{2}}=a_{x_{1}}+a_{x_{2}} .
$$

Now we claim that, for any $x \in A_{d} \backslash\{z\}$,

$$
D_{x} \partial=a_{x} \partial, \forall \partial \in T_{x-z} .
$$


If $\hat{x} \in F \hat{z}$, this follows from (4.8') since $T_{x} \cap T_{z}=T_{x} \cap T_{z}=T_{z}$. If $x \in A_{d} \backslash\left(A_{d}^{+} \cup\{z\}\right),(4.24)$ is clear. Next we suppose $x \in A_{d}^{+}$with $\hat{x} \notin F \hat{z}$. We shall show this by induction on $d(x):=\sum_{i \in I} d_{i}(x)$.

If $d(x)=0$, by replacing $D$ with $D+D_{\mu}$ for a suitable $\mu \in \operatorname{hom}(A, F)$, we may assume that $a_{x} \neq 0$. For $\partial \in T_{x-z} \backslash T_{z}$ let $D\left(t^{x} \partial\right)=a_{x} t^{x} \partial^{\prime}$. For any $i \in I, u_{i} \in A_{i}$, appling $D$ to $\left[t^{x} \partial, t^{u_{i}} d_{i}\right]=t^{x+u_{i}} \partial\left(u_{i}\right) d_{i}$, we obtain that

$$
\left[a_{x} t^{x} \partial^{\prime}, t^{u_{i}} d_{i}\right]+\left[t^{x} \partial, a_{u_{i}} t^{u_{i}} d_{i}\right]=\left(a_{x}+a_{u_{i}}\right) t^{x+u_{i}} \partial\left(u_{i}\right) d_{i} .
$$

We deduce that $a_{x}\left(\partial-\partial^{\prime}\right)\left(u_{i}\right) d_{i}=0$. Thus $\partial\left(u_{i}\right)=\partial^{\prime}\left(u_{i}\right)$ for all $u_{i} \in A_{i}$ and any $i \in I$. So we obtain that $\partial=\partial^{\prime}$.

Suppose (4.24) holds for any $x \in A_{d} \backslash\{z\}$ with $d(x) \leq k$ where $k \geq 0$. Consider $x_{1} \in A_{d}^{+} \backslash(\mathbf{Z} z)$ with $d\left(x_{1}\right)=k+1$. Suppose $t^{x_{1}} \partial \in\left(S_{d}\right)_{x_{1}}$ with $\partial \in T_{x-z} \backslash T_{z}$. For any $i \in I$ and any $u_{i} \in A_{i}$, we know that $\left.D\right|_{\left(S_{d}\right)_{x_{1}+u_{i}}}=$

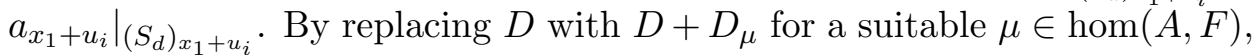
we may assume that $a_{x_{1}} \neq 0$. Let $D\left(t^{x_{1}} \partial\right)=a_{x_{1}} t^{x_{1}} \partial^{\prime}$. Appling $D$ to $\left[t^{x_{1}} \partial, t^{u_{i}} d_{i}\right]=t^{x_{1}+u_{i}}\left(\partial\left(u_{i}\right) d_{i}-d_{i}\left(x_{1}\right) d_{i}\right)$, we obtain that

$$
a_{x_{1}}\left[t^{x_{1}} \partial^{\prime}, t^{u_{i}} d_{i}\right]+\left[t^{x_{1}} \partial, a_{u_{i}} t^{u_{i}} d_{i}\right]=\left(a_{x_{1}}+a_{u_{i}}\right) t^{x_{1}+u_{i}}\left(\partial\left(u_{i}\right) d_{i}-d_{i}\left(x_{1}\right) d_{i}\right) .
$$

Then we infer that $d_{i}\left(x_{1}\right)\left(\partial-\partial^{\prime}\right)=\left(\partial-\partial^{\prime}\right)\left(u_{i}\right) d_{i}$. If $\left(\partial-\partial^{\prime}\right)\left(u_{i}\right) \neq 0$, we deduce that $d_{i} \in T_{x_{1}-z}$. This contradicts the fact that $x_{1} \in A_{d}^{+}$. So we deduce that $\partial\left(u_{i}\right)=\partial^{\prime}\left(u_{i}\right)$ for all $u_{i} \in A_{i}$, any $i \in I$. Thus $\partial=\partial^{\prime}$.

By induction we see that (4.24) is true. Define $\nu \in \operatorname{hom}(A, F)$ so that $\nu(x)=a_{x}$ for all $x \in A_{d}^{+}$. Then we see that $\left.D\right|_{S_{d}^{+}}=$ad $\left.\left(t^{y} \partial_{0}\right)\right|_{S_{d}^{+}}$. By Lemma 4.5 we conclude that $D=D_{\nu}$.

We now summarize the results on derivations of $S_{d}(A, T, \varphi, z)$ obtained in this section.

Theorem 4.9. Every $D \in \operatorname{Der}\left(S_{d}(A, T, \varphi, z)\right)$ has the form $D=\sum_{y \in A} D_{y}$ for degree $y$ derivations $D_{y}$, such that for each $u \in S_{d}(A, T, \varphi, z)$ there only finitely many $y \in A$ with $D_{y}(u) \neq 0$, where

(a) $D_{y}=\operatorname{ad}\left(t^{y} \partial_{0}\right)$ for some $t^{y} \partial_{0} \in\left(S_{d}\right)_{y}$ if $y \in A_{d} \backslash\{0, z\}$;

(b) $D_{y}=a$ ad $\left(t^{y} d_{1}\right)$ for some $a \in F$ if $y=z \in A_{d}$;

(c) $D_{y}=D_{\nu}$ for some $\nu \in \operatorname{hom}(A, F)$ if $y=0$.

As in [3, Proposition 3.3], we also have that the sum $D=\sum_{y \in A} D_{y}$ in the above Theorem is finite if $\operatorname{dim} T<\infty$.

\section{References}

[1] A.A. Albert and M.S. Frank, Simple Lie algebras of characteristic p, Univ. e Politec. Torino Rend. Sem. Mat., 14 (1954-55), 117-139.

[2] R. Block, On torsion-free abelian groups and Lie algebras, Proc. Amer. Math. Soc., 9 (1958), 613-620. 
[3] D. Ž. Đoković and K. Zhao, Derivations, isomorphisms, and second cohomology of generalized Witt algebras, Trans. Amer. Math. Soc., 350(2) (1998), 643-664.

[4] _ Derivations, isomorphisms, and second cohomology of generalized Block algebras, Algebra Colloquium, 3 (1996), 245-272.

[5] _ Generalized Cartan type W Lie algebras in characteristic 0, J. Alg., 195 (1997), 170-210.

[6] __ Some infinite dimensional simple Lie algebras in characteristic 0 related to those of Block, J. Pure and Applied Algebras, 127(2) (1998), 153-165.

[7] _ Generalized Cartan type S Lie algebras in characteristic 0, J. Alg., 193 (1997), 144-179.

[8] _ Some infinite dimensional simple subalgebras of generalized Block algebras, J. Alg., 192 (1997), 74-101.

[9] _ Second cohomology of generalized Cartan type $S$ Lie algebras in characteristic zero, J. Pure and Applied Algebras, 136 (1999), 101-126.

[10] I. Kaplansky, Seminar on simple Lie algebras, Bull. Amer. Math. Soc., 60 (1954), 470-471.

[11] N. Kawamoto, Generalizations of Witt algebras over a field of characteristic zero, Hiroshima Math. J., 16 (1986), 417-426.

[12] J.M. Osborn, New simple infinite dimensional Lie algebras of characteristic 0, J. Alg., 185 (1996), 820-835.

[13] _ Derivations and isomorphisms of Lie algebras of characteristic 0, Studies in Advanced Mathematics, 4 (1997), 95-108.

[14] A.N. Rudakov, Group of automorphisms of infinite-dimensional simple Lie algebras, Math. USSR-Izvestija, 3(4) (1965), 707-722.

[15] I.M. Singer and S. Sternberg, The infinite groups of Lie and Cartan, I: The transitive groups, J. Analyse Math., 15 (1965), 1-114.

Received August 19, 1997 and revised February 23, 1999. This author was supported by NSF of China.

\author{
Academia Sinica \\ BEIJING, 100080 \\ CHINA \\ E-mail address: zhao@iss06.iss.ac.cn \\ UNIVERSITY OF WISCONSIN-MADISON \\ MADISON, WI 53706
}

